\title{
Effects of PI3K and FGFR inhibitors alone and in combination, and with/without cytostatics in childhood neuroblastoma cell lines
}

\author{
STEFAN HOLZHAUSER ${ }^{1}$, MONIKA LUKOSEVICIUTE ${ }^{1}$, CHRISTINA PAPACHRISTOFI $^{1}$, \\ CHRISTINA VASILOPOULOU ${ }^{1}$, NIKOLAS HEROLD ${ }^{2,3}$, MALIN WICKSTRÖM ${ }^{2}$, \\ OURANIA N. KOSTOPOULOU ${ }^{1 *}$ and TINA DALIANIS ${ }^{1 *}$
}

Departments of ${ }^{1}$ Oncology-Pathology, and ${ }^{2}$ Children and Women's Health, Karolinska Institutet;

${ }^{3}$ Department of Paediatric Oncology, Theme of Children's Health, Karolinska University Hospital, 17164 Stockholm, Sweden

Received September 23, 2020; Accepted December 15, 2020

DOI: $10.3892 /$ ijo.2021.5167

\begin{abstract}
Neuroblastoma (NB) is a heterogenous disease with treatment varying from observation for low-risk tumors, to extensive therapy with chemotherapy, surgery,radiotherapy, and autologous bone-marrow-transplantation and immunotherapy. However, a high frequency of primary-chemo-refractory disease and recurrences urgently require novel treatment strategies. The present study therefore investigated the anti-NB efficacy of the recently FDA-approved phosphoinositide 3-kinase (PI3K) and fibroblast growth factor receptor (FGFR) inhibitors, alpelisib (BYL719) and erdafitinib (JNJ-42756493), alone and in combination with or without cisplatin, vincristine, or doxorubicin on $5 \mathrm{NB}$ cell lines. For this purpose, the NB cell lines, SK-N-AS, SK-N-BE(2)-C, SK-N-DZ, SK-N-FI and SK-N-SH (where SK-N-DZ had a deletion of PIK3C2G and none had FGFR mutations according to the Cancer Program's Dependency Map, although some were chemoresistant), were tested for their sensitivity to FDA-approved inhibitors alone or in combination, or together with cytostatic drugs by viability, cytotoxicity, apoptosis and proliferation assays. The results revealed that monotherapy with alpelisib or erdafitinib resulted in a dose-dependent inhibition of cell viability and proliferation. Notably, the combined use of PI3K and FGFR inhibitors resulted in an enhanced efficacy, while their combined use with the canonical cytotoxic agents, cisplatin, vincristine and doxorubicin, resulted in variable synergistic, additive and antagonistic effects. Collectively, the present study provides
\end{abstract}

Correspondence to: Dr Ourania N. Kostopoulou or Professor Tina Dalianis, Department of Oncology-Pathology, Karolinska Institutet, Bioclinicum J6:20, 17164 Stockholm, Sweden

E-mail: ourania.kostopoulou@ki.se

E-mail: tina.dalianis@ki.se

*Contributed equally

Key words: neuroblastoma, PI3K inhibitors, FGFR inhibitors, chemotherapy, cisplatin, vincristine, doxorubicin, BYL719, JNJ-42756493 pre-clinical evidence that PI3K and FGFR inhibitors exhibit promising anti-NB activity. The data presented herein also indicate that the incorporation of these inhibitors into chemotherapeutic regimens requires careful consideration and further research in order to obtain a beneficial efficacy. Nevertheless, the addition of PI3K and FGFR inhibitors to the treatment arsenal might reduce the occurrence of refractory and relapsing disease in NB without FGFR and PI3K mutations.

\section{Introduction}

Neuroblastoma (NB), is an embryonal tumor derived from precursors of the sympathetic peripheral nervous system with heterogenous biology and genetics, as well as diverse clinical presentation, ranging from spontaneous regression to aggressive progressive metastatic disease (1). It is also the most common solid extracranial tumor in children (1). While cure rates for low- and intermediate risk NB are $>90 \%$, high-risk NB exhibits a 5-year overall survival of only 40-50\%, despite highly aggressive multimodal therapy with multiagent chemotherapy, surgery, radiotherapy, high-dose chemotherapy with autologous bone-marrow-transplantation and immunotherapy $(1,2)$. Apart from treatment-related mortality, this is mainly due to the fact that primary refractory, or relapsed NB responds poorly to salvage chemotherapy and radiotherapy $(1,3)$. Therefore, there is an urgent medical need for novel treatment strategies to: i) Reduce the incidence of refractory and recurring NB; and ii) increase the therapeutic efficacy of salvage treatments.

A subset of NB harbors somatic or germ-line mutations of anaplastic lymphoma kinase (ALK), a gene recurrently mutated or rearranged in particular in adenocarcinomas of the lung, the colon and the breast, as well as in a number of other types of cancer, including NB (4). The prevalence of ALK alterations is up to $14 \%$ in high-risk NB, and the association of ALK with a poor survival suggests that if functions as an oncogenic driver in NB (5). Even though the emergence of resistance is a concern, ALK inhibitors have exhibited promising efficacy in individual patients (6). Hence, the targeting of specific oncogenic pathways appears to be a promising bona fide strategy for NB. 
Fibroblast growth factor receptors (FGFRs), a family of tyrosine kinase receptors not extensively studied in childhood cancer, are recurrently mutated and deregulated in adult cancers, and both unspecific and specific FGFR inhibitors targeting these genes have been developed $(7,8)$. Likewise and at an even higher frequency, members of the phosphoinositide 3 kinase (PI3K) family are dysregulated in a number of types of cancer, including childhood cancer, and here as well, several PI3K inhibitors with varying specificity with respect to different subunits, have been developed (9-12); several of these inhibitors have been evaluated in clinical trials (13).

Recently, the authors examined 29 NB patient tumor samples for possible mutations in phosphatidylinositol-4,5-bisphosphate 3-kinase, catalytic subunit alpha (PIK3CA), as well as in FGFR3 (14). It was found that these were not commonly occurring; however, one FGFR mutation was identified in one of the 29 patients with NB (14). Nonetheless, it was revealed that the well-established NB cell lines, SK-N-AS, SK-N-BE(2)-C (MYCN amplified and chemotherapy-resistant), SK-N-DZ (MYCN amplified and a frameshift deletion of PIK3C2G), SK-N-FI and SK-N-SH (the only cell line with wild-type TP53), exhibited dose dependent responses to both PI3K (BKM120 and BEZ235) and FGFR (AZD4547) inhibitors (14). Furthermore, combining the 2 types of inhibitors yielded more potent synergy. Given the biological heterogeneity of NB cell lines tested with respect to FGFR or PI3K mutations, MYCN amplifications, 11q deletions and sensitivity to chemotherapy, the data suggest that NB is broadly vulnerable to FGFR and PI3K inhibition (14-19).

While our recent studies were based on inhibitors at a pre-clinical or early clinical trial stage, the FDA has recently approved the PI3K inhibitor, alpelisib (BYL719), and the FGFR inhibitor, erdafitinib (JNJ-42756493) (14,20-22). The former is approved for certain types of breast cancer, preferentially with PI3K mutations, while the other is used for specific solid tumors, mainly with FGFR mutations, or chromosomal rearrangements $(21,22)$. Notably, however, erdafitinib is currently tested in the pediatric MATCH phase II clinical trial, including recurrent/relapsed neuroblastoma with FGFR mutations (ClinicalTrials. gov, identifier: NCT03210714 and NCT03155620). As approved drugs could directly be used for the treatment of NB in for example, a compassionate use setting, the present study aimed to investigate the anti-NB effects of alpelisib and erdafitinib alone and in combination, as well as in combination with standard NB cytotoxic drugs.

\section{Materials and methods}

Tumor cell lines, culture conditions and cell seeding. The five 5 cell lines, SK-N-AS, SK-N-BE(2)-C, SK-N-DZ, SK-N-FI and SK-N-SH, were used for the in vitro experiments and were kindly provided by Professor Per Kogner, Karolinska Institutet (15-19). Short tandem repeat genetic profiling using the AmpFLSTR Identifiler PCR Amplification kit (Applied Biosystems) in 2016 was performed to verify the identities of the cell lines. None of the cell lines used in the present study had any FGFR3 mutations according to the Cancer Dependency Map (https://depmap.org/portal/), while only SK-N-DZ had a frameshift deletion of PIK3C2G. The SK-N-DZ and SK-N-BE(2)-C cells are MYCN-amplified and only the SK-N-SH cell line is TP53 wild-type. Some characteristics of the cell lines are summarized in Table SI. The SK-N-BE(2)-C cell line was derived from a previously treated relapsed patient and is known to be chemoresistant, for example to doxorubicin (18).

Roswell Park Memorial Institute (RPMI; Gibco; Thermo Fisher Scientific, Inc.), supplemented with 10\% FBS (fetal bovine serum; Gibco; Thermo Fisher Scientific, Inc.), 1\% L-glutamine, $100 \mathrm{U} / \mathrm{ml}$ of penicillin and $100 \mu \mathrm{g} / \mathrm{ml}$ streptomycin was used for the culture of all cell lines, and the cells were maintained at $37^{\circ} \mathrm{C}$ in a humidified incubator with $5 \% \mathrm{CO}_{2}$.

In all assays, 5,000 cells were seeded in 90-200 $\mu 1$ medium/well (without penicillin and streptomycin to avoid any interference with our drugs) in 96-well plates, and the edges were filled with medium to avoid edge effects.

\section{Inhibitor and cytostatic treatment}

PI3K and FGFR inhibitors. The PI3K inhibitors, dactolisib (BEZ235, NVP-BEZ235) and alpelisib (BYL719), and the FGFR inhibitors, AZD4547 and JNJ-42756493 (erdafitinib), used in the present study were all purchased from Selleckchem Chemicals. Dimethyl sulfoxide (DMSO; Sigma-Aldrich; Merck KGaA) was used for the stock dilutions, which were diluted further with PBS for the intended concentrations. The cells were treated with the inhibitors $24 \mathrm{~h}$ after seeding and the dose ranges used were as follows: AZD4547, 5.0-25 $\mu \mathrm{M}$; JNJ-42756493, 0.01-10 $\mu \mathrm{M}$; BEZ235, 0.25-5.0 $\mu \mathrm{M}$; and BYL719, 0.25-10 $\mu \mathrm{M}$.

Cytostatics. The cytostatics used for the current experiment were as follows: Cisplatin (Accord Healthcare Ltd.), vincristine (Oncovin, Pfizer) and doxorubicin (Accord Healthcare Ltd.). All the cytostatic stock solutions were diluted in PBS and further diluted in PBS prior to each experiment, and used at the following concentrations: Cisplatin, 0.1-40 $\mu \mathrm{M}$; vincristine, 0.001-1 $\mu \mathrm{M}$; and doxorubicin, 0.1-5 $\mu \mathrm{M}$.

WST-1 viability assay. A WST-1 assay (Roche Diagnostics) was used to measure cell viability, which was followed for up to $72 \mathrm{~h}$ after seeding according to a previously described protocol (20).

\section{Proliferation, cell cytotoxicity and apoptosis assays}

Proliferation assays. Cells were seeded in $200 \mu \mathrm{l}$ medium/well in a 96-well plate and were placed into the IncuCyte S3 Live-Cell Analysis System (Essen Bioscience) for up to $72 \mathrm{~h}$ after seeding. The machine was set to scan the plates and obtain images every $2 \mathrm{~h}$. PBS was used as a control and culture medium was used as the background. Cell proliferation was observed by analyzing the confluence of cell in the images (20).

Cell cytotoxicity and apoptosis assays. IncuCyte Red Cytotoxicity reagent and IncuCyte Caspase-3/7 Green Apoptosis assay (both from Essen Bioscience) were used to measure cytotoxicity and apoptosis, respectively. At $24 \mathrm{~h}$ after seeding, the medium was discarded and replaced with fresh medium, which contained the cytotoxicity reagent (final concentration of $250 \mathrm{nM}$ per well) and the apoptosis reagent at a ratio of 1:1,000. Subsequently, the indicated inhibitors or 

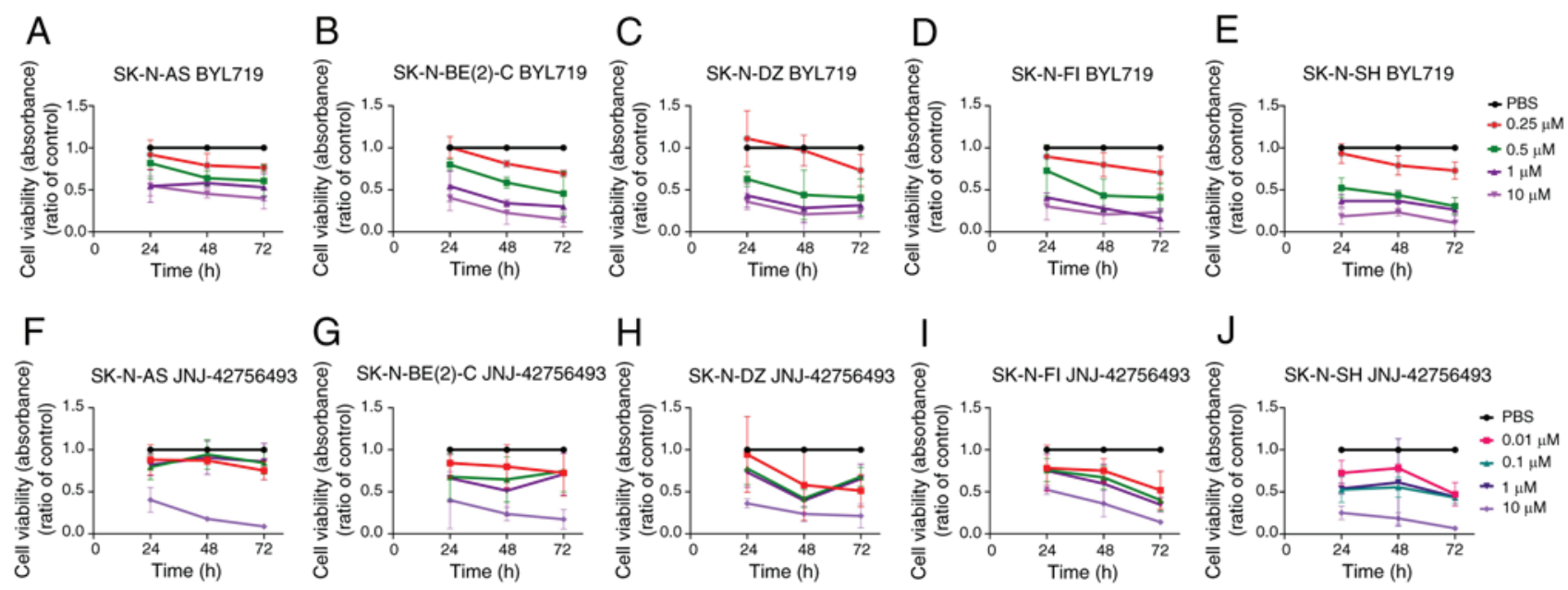

Figure 1. WST-1 viability assays on SK-N-AS, SK-N-BE(2)-C, SK-N-DZ, SK-N-FI and SK-N-SH cell lines upon treatment with the PI3K inhibitor, BYL719, and FGFR inhibitor, JNJ-42756493. WST-1 viability assay measured the absorbance following treatment for 24, 48 and $72 \mathrm{~h}$ of SK-N-AS, SK-N-BE(2)-C, SK-N-DZ, SK-N-FI and SK-N-SH cells with (A-E) PI3K inhibitor, BYL719, and (F-J) FGFR inhibitor, JNJ-42756493. The graphs represent 3 experimental runs per cell line and results are presented as the means \pm standard deviation.

chemotherapeutic agents were added either alone or combined and simultaneously as indicated below. The plates were then incubated at $37^{\circ} \mathrm{C}$ for up to $72 \mathrm{~h}$ following treatment in the IncuCyte S3 Live-Cell Analysis System (Essen Bioscience), where the machine obtained images every $2 \mathrm{~h}$ [further details regarding this assay have been previously described (20)].

Statistical analysis. The effects of treatments (single or combined) were analyzed by a multiple t-test accompanied by a correction for multiple comparison of the means conferring to the Holm-Sidak method was performed. The 'Highest Single Agent' and dose-effect-based approach 'median-effect method' (based on Loewe Additivity) approach were used to analyze the combinational effects of the drugs $(23,24)$. This method describes whether the achieved effect of a drug combination $(\mathrm{EAB})$ is larger than the effects obtained by any of the individual drugs (EA and EB). A combination index (CI) was determined using the following formula: $\mathrm{CI}=\max (\mathrm{EA}$, EB)/EAB. A CI $<1$ was demarcated as a positive combination effect and CI $>1$ as a negative combination effect. Another method that we used to analyze the combinational effect was the median-effect method of Chou (Chou-Talalay method) (24) by using ComboSyn software (http://www.combosyn.com; ComboSyn, Inc.). The dose-response curves were fitted to a linear model using the median-effect equation, allowing for the calculation of a median-effect value D (equivalent to IC50) and slope. Goodness-of-fit was assessed with the linear correlation coefficient, $r$; $r>0.85$ was required for the analysis to be approved. The degree of drug interaction was rated using the $C I$ for mutually exclusive drugs: $\mathrm{CI}=\mathrm{d} 1 / \mathrm{D} 1+\mathrm{d} 2 / \mathrm{D} 2$, where D1 and D2 represent the concentration of drug 1 and 2 alone, respectively, that is required to produce a certain effect, and $\mathrm{d} 1$ and $\mathrm{d} 2$ represent the concentration of drugs 1 and 2 in combination that is required to produce the same effect. $\mathrm{CI}<0.70$ was defined as synergy and $\mathrm{CI}>1.45$ as antagonism, and values in between as additive effects, according to the recommendations of the ComboSyn software. One-way ANOVA with the Bonferroni post hoc test was utilized to analyze the difference in means between the 2 single drugs and the combinational treatment. A P $<0.05$ was considered to indicate a statistically significant difference.

\section{Results}

Effects following single-drug exposure of $S K-N-A S$, $S K-N-B E(2)-C, S K-N-D Z, S K-N-F I$ and $S K-N-S H$ cells to PI3K and FGFR inhibitors. All NB lines exhibited dose-dependent responses to the PI3K inhibitor, BYL719 $(0.25-10 \mu \mathrm{M})$, and the FGFR inhibitor, JNJ-42756493 (0.01-10 $\mu \mathrm{M})$, compared to treatment with PBS, as shown by WST-1 assays, assessing cellular metabolic capacity colorimetrically (viability/proliferation/cytotoxicity) by absorbance. Data summarizing 3 experiments per NB cell line with BYL719 and JNJ-42756493 at up to $72 \mathrm{~h}$ after treatment are presented in Fig. 1. IC50 values from dose response analysis for BYL719 and JNJ-42756493 for 24, 48 and $72 \mathrm{~h}$ are presented in Table I. These assays were subsequently complemented for BYL719 and JNJ-42756493, with proliferation, cytotoxicity and apoptosis assays presented below. Corresponding data were reported before for the PI3K inhibitor, BEZ235, and the FGFR inhibitor, AZD4547 (14).

BYL719. All NB lines presented decreased viability compared to PBS early on following treatment with the majority of the BYL719 concentrations used (for all at least $\mathrm{P}<0.05$ ), except at the concentration of $0.25 \mu \mathrm{M}$ BYL719 for all cell lines at 24 and $48 \mathrm{~h}$, and apart from the SK-N-BE(2)-C cells at $48 \mathrm{~h}$, and with $0.5 \mu \mathrm{M}$ BYL719 at $24 \mathrm{~h}$ for the SK-N-FI and SK-N-AS cells (Fig. 1A-E).

$J N J-42756493$. The concentration of $10 \mu \mathrm{M} \mathrm{JNJ}-42756493$ significantly decreased the viability of all NB cell lines compared to PBS treatment early on following treatment at all recorded time points (for all, at least $\mathrm{P}<0.05$ ) (Fig. 1F-J). This was also observed at the concentrations of $0.1-1 \mu \mathrm{M}$ JNJ-42756493 for the SK-N-FI cells (Fig. 1I), and with the concentrations of 0.01-1 $\mu \mathrm{M}$ JNJ-42756493 for the SK-N-SH 
Table I. Estimation of IC50 values based on WST-1 viability analysis following treatment with the FGFR inhibitor, JNJ-42756493, PI3K inhibitor, BYL719, and the cytostatic drugs cisplatin, vincristine and doxorubicin for 24,48 and $72 \mathrm{~h}$.

\begin{tabular}{|c|c|c|c|c|}
\hline \multirow[b]{2}{*}{ Drugs } & \multirow[b]{2}{*}{ Cell lines } & \multicolumn{3}{|c|}{$\mathrm{IC50}(\mu \mathrm{M})$} \\
\hline & & $24 \mathrm{~h}$ & $48 \mathrm{~h}$ & $72 \mathrm{~h}$ \\
\hline \multirow[t]{5}{*}{ BYL } & SK-N-AS & 4.78 & 1.56 & 1.14 \\
\hline & SK-N-BE(2)-C & 2.56 & 0.74 & 0.48 \\
\hline & SK-N-DZ & 1.42 & 0.66 & 0.49 \\
\hline & SK-N-FI & 1.19 & 0.52 & 0.38 \\
\hline & SK-N-SH & 0.79 & 0.60 & 0.38 \\
\hline \multirow[t]{5}{*}{ JNJ } & SK-N-AS & 5.93 & 3.73 & 2.69 \\
\hline & SK-N-BE(2)-C & 3.38 & 0.84 & 1.99 \\
\hline & SK-N-DZ & 3.93 & 0.05 & 1.63 \\
\hline & SK-N-FI & 8.48 & 1.85 & 0.03 \\
\hline & SK-N-SH & 0.72 & 0.90 & 0.02 \\
\hline \multirow[t]{5}{*}{ CIS } & SK-N-AS & 12.64 & 1.50 & 0.78 \\
\hline & SK-N-BE(2)-C & 12.08 & 8.10 & 3.07 \\
\hline & SK-N-DZ & 36.51 & 8.92 & 0.20 \\
\hline & SK-N-FI & $74.5^{\mathrm{a}}$ & 9.54 & 7.97 \\
\hline & SK-N-SH & 4.28 & 2.13 & 0.58 \\
\hline \multirow[t]{5}{*}{ VIN } & SK-N-AS & $2.47^{\mathrm{a}}$ & 0.006 & 0.003 \\
\hline & SK-N-BE(2)-C & 0.33 & 0.07 & 0.07 \\
\hline & SK-N-DZ & 0.86 & 0.03 & 0.03 \\
\hline & SK-N-FI & 1.13 & $<0.001^{\mathrm{b}}$ & 0.001 \\
\hline & SK-N-SH & 0.68 & 0.002 & $<0.001^{\mathrm{b}}$ \\
\hline \multirow[t]{5}{*}{ DOXO } & SK-N-AS & 7.69 & 0.77 & 0.15 \\
\hline & SK-N-BE(2)-C & 3.54 & 0.71 & 0.17 \\
\hline & SK-N-DZ & 4.12 & 0.36 & 0.09 \\
\hline & SK-N-FI & 3.18 & 0.71 & 0.35 \\
\hline & SK-N-SH & 0.38 & 0.09 & 0.04 \\
\hline
\end{tabular}

The inhibitory concentration $50 \%$ (IC50) for each cell line for each drug was determined from log concentrations effect curves in GraphPad Prism using non-linear regression analysis. ${ }^{\text {aExtrapolated }}$ IC50 value, i.e., outside the tested concentration range. ${ }^{\text {b}}$ The IC50 value could not be determined; lowest/highest tested concentration closest to the IC50 is reported.

cells (Fig. 1J) (for all, at least $\mathrm{P}<0.05$ ), while the SK-N-AS and SK-N-BE(2)-C cells tended to be more resistant.

To conclude, all 4 NB lines exhibited dose-dependent responses to all inhibitors, with IC50 values ranging from 0.38 to $4.78 \mu \mathrm{M}$ for BYL719 and 0.02 to $8.48 \mu \mathrm{M}$ for JNJ-42756493, with the SK-N-SH cells generally being more sensitive, and the SK-N-AS cells generally being more resistant to both BYL719 and JNJ-42756493 (Table I). Notably, NB cell lines with high-risk genetic alterations, such as MYCN amplification, were in general not less sensitive to the FGFR and PI3K inhibitors.

Effects following combined exposure of $S K-N-A S$, $S K-N-B E(2)-C, S K-N-D Z, S K-N-F I$ and $S K-N-S H$ cells to $P I 3 K$ and FGFR inhibitors. All NB lines were treated with combinations of the PI3K inhibitors, BYL719 $(0.25-1 \mu \mathrm{M})$ and BEZ235 (0.25-1 $\mu \mathrm{M})$, and the FGFR inhibitors, JNJ-42756493 (0.01-0.1 $\mu \mathrm{M})$ and AZD4547 (5-10 $\mu \mathrm{M})$, and assessed by WST-1 assays, since the combination of BEZ235 and AZD4547 has previously shown synergy (14). In addition, under these conditions, an enhanced efficacy was observed, despite omitting the previously used highest concentration of the inhibitors in the combination experiments. Data from 3 experiments with the FDA-approved BYL719 and JNJ-42756493, as well as additional combinations, including BEZ235 and AZD4547 with a read out of $72 \mathrm{~h}$ following treatment are presented in Fig. 2.

BYL719 and JNJ-42756493. All NB lines exhibited a significantly decreased absorbance compared to PBS at all time points examined, with the highest $1 \mu \mathrm{M}$ BYL719 and $0.1 \mu \mathrm{M}$ JNJ-42756493 combination (for all, at least $\mathrm{P}<0.05$ ) (Fig. 2A-E). SK-N-SH was the most sensitive cell line with a significantly lower absorption compared to PBS with all combinations at all time points examined (at least, $\mathrm{P}<0.05$ ) (Fig. 2E). The other NB lines also exhibited a decreased absorbance compared to PBS with the lower concentrations of BYL719 and JNJ-42756493 used, but did not reach statistical significance at all time points (Fig. 2A-D).

BYL719 and AZD4547, as well as, BEZ235 and JNJ-42756493. The FDA-approved inhibitors were also combined with the previously tested inhibitors. A decreased absorbance compared to PBS was observed for all NB lines at all time points with all concentrations used, apart from the SK-N-DZ cells at $24 \mathrm{~h}$ following treatment with $0.25 \mu \mathrm{M}$ of BYL719 and $5 \mu \mathrm{M}$ AZD4547, and for the SK-N-AS cells at $48 \mathrm{~h}$ following treatment with $0.25 \mu \mathrm{M}$ BEZ235 and $0.01 \mu \mathrm{M} \mathrm{JNJ}-42756493$ (for all others, at least $\mathrm{P}<0.05$ ) (Fig. 2F-O).

Combinational effect analysis and the dose-effect-based median-effect-principle were also calculated as described below $(23,24)$ and the findings are presented below. Briefly, for the combinational effect analysis having a combinatorial index (CI) $\mathrm{CI}<1$ indicates a positive and a CI $>1$ a negative effect. For the dose-effect-based median-effect principle, a CI $<0.75$ indicates synergism, a $\mathrm{CI}>0.75$, but $<1.45$ indicates an additive effect, while a CI $>1.45$ indicates an antagonistic effect.

The CIs for the BYL719 and JNJ-42756493, BEZ235 and AZD4547, BYL719 and AZD4547, as well as the BEZ235 and JNJ-42756493 combinations were calculated at 24 and $48 \mathrm{~h}$ following treatment, and the CIs after $24 \mathrm{~h}$ are presented in Fig. 3. Data [for BEZ235 and AZD4547 data were in line to what has been previously reported earlier (14)]. The overall combination effect was positive and the majority of the drug combinations indicated a positive effect $(\mathrm{CI}<1$, i.e., an improved combinational effect on viability than the best single drug) for the majority of the NB cell lines (Fig. 3). The SK-N-BE(2)-C and SK-N-DZ cells were less sensitive to some concentration combinations of BYL719 and JNJ-42756493, and BEZ235 and JNJ-42756493, compared to the other NB cell lines (Fig. 3). Furthermore, while the SK-N-AS cell line was sensitive to the BYL719 and JNJ-42756493 combination, it tended over time to be less sensitive to the BYL719 and AZD4547, and BEZ235 and JNJ-42756493 combinations (Fig. 3 and data not shown). To summarize, the majority of the PI3K and FGFR inhibitor combinations exerted additive or synergistic effects on all NB 
A

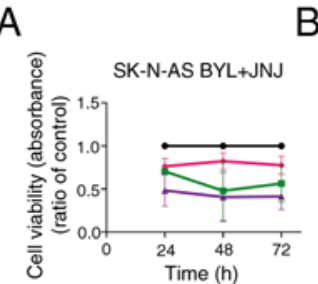

$\mathrm{B}$

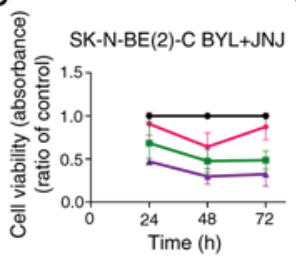

C

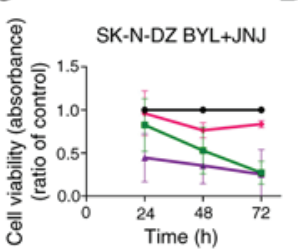

D

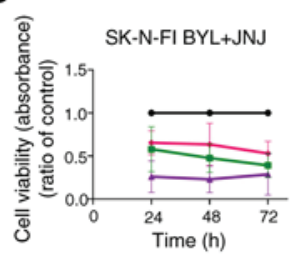

E

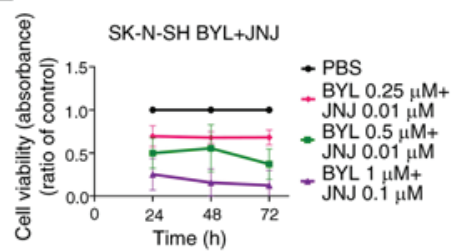

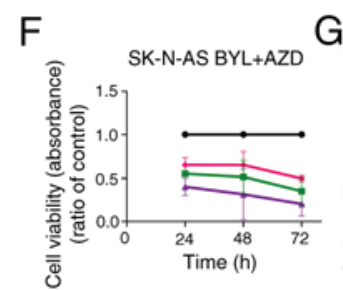
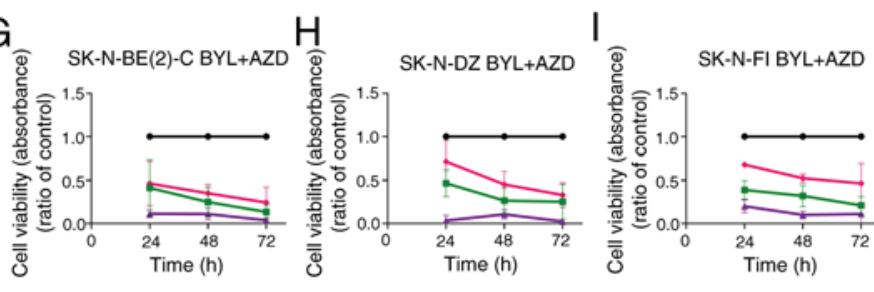

J
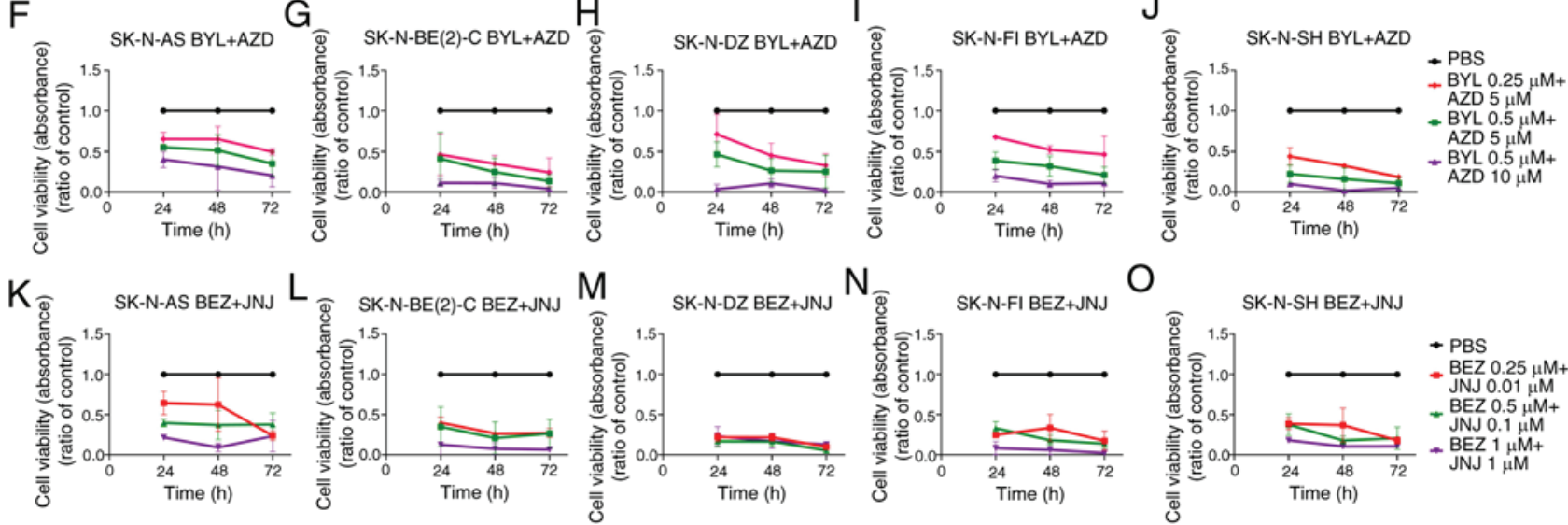

○

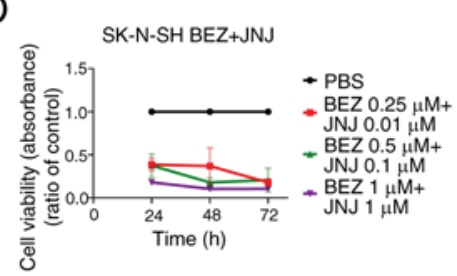

Figure 2. WST-1 viability assays on SK-N-AS, SK-N-BE(2)-C, SK-N-DZ, SK-N-FI and SK-N-SH cell lines following combined treatments with PI3K inhibitors (BYL719, BEZ235) and FGFR inhibitors (JNJ-42756493, AZD4547). WST-1 viability assay measured the absorbance following treatment for 24,48 and $72 \mathrm{~h}$ of SK-N-AS, SK-N-BE(2)-C, SK-N-DZ, SK-N-FI and SK-N-SH cells with (A-E) BYL719 and JNJ-42756493, (F-J) BYL719 and AZD4547, and (K-O) BEZ235 and JNJ-42756493. The graphs represent 3 experimental runs per cell line and results are presented as the means \pm standard deviation. BYL, BYL719; JNJ, JNJ-42756493; BEZ, BEZ235; AZD, AZD4547.

A
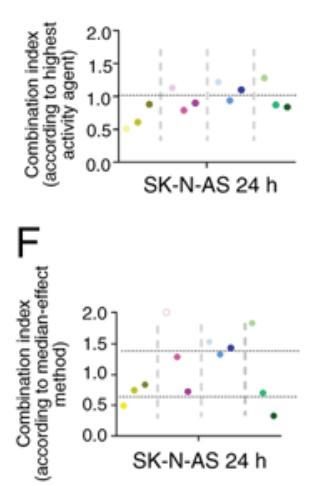

Shows samples $\mathrm{Cl}>2$

$\times$ Shows not applicabel $\mathrm{Cl}$
B

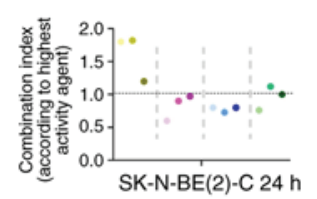

G

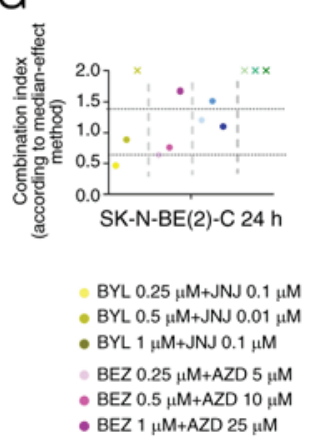

C

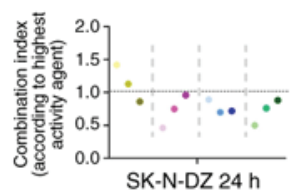

$\mathrm{H}$

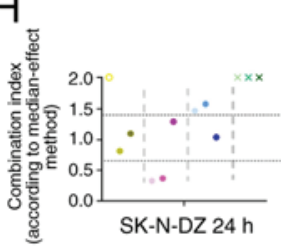

BYL $0.25 \mu \mathrm{M}+\mathrm{AZD} 5 \mu \mathrm{M}$

- BYL $0.5 \mu \mathrm{M}+\mathrm{AZD} 5 \mu \mathrm{M}$
$-\mathrm{BYL} 0.5 \mu \mathrm{M}+\mathrm{AZD} 10 \mu \mathrm{M}$

- BEZ $0.25 \mu \mathrm{M}+\mathrm{JNJ} 0.01 \mu \mathrm{M}$

- BEZ $0.5 \mu \mathrm{M}+\mathrm{JNJ} J 0.1 \mu \mathrm{M}$

- BEZ $1 \mu \mathrm{M}+\mathrm{JNJ} 1 \mu \mathrm{M}$
D

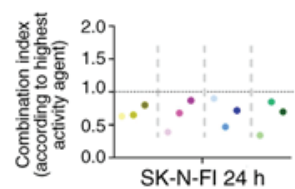

I

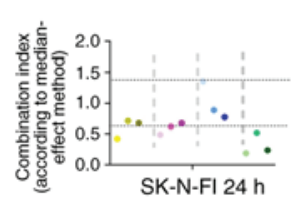

E

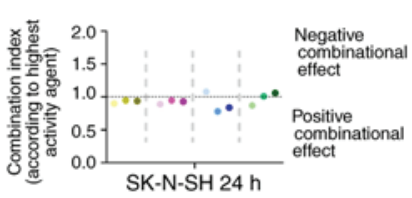

$\mathrm{J}$

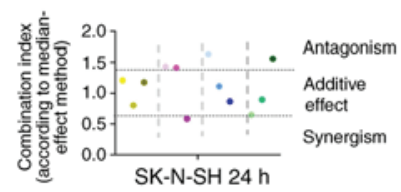

Figure 3. Combinational effects of PI3K inhibitors, BYL719 and BEZ235, and FGFR inhibitors, JNJ-42756493 and AZD4547, on SK-N-AS, SK-N-BE(2)-C, SK-N-DZ, SK-N-FI and SK-N-SH cell lines. CIs were obtained by (A-E) the highest single agent approach, where CI $>1$ shows a negative combination effect, and (F-J) the median effect method, where $\mathrm{CI}>1.45$ indicates antagonism, $0.7<\mathrm{CI}>1.45$ additive, and $\mathrm{CI}<0.7$ synergistic combinational effects. $\mathrm{CIs}$ were calculated from the mean of 3 experiments analyzed by WST 1, at $24 \mathrm{~h}$ following treatment. $\mathrm{x}$ denotes $\mathrm{r}<0.85$ in the median method; thus, the analysis could not proceed; o denotes CI >2, which indicates a negative combination effect. CI, combination index; NB, neuroblastoma; BYL, BYL719; BEZ, BEZ235; AZD, AZD4547; JNJ, JNJ-42756493.

lines, with the former being useful to avoid resistance, and the latter for dose reduction.

Effects of single cisplatin, vincristine or doxorubicin treatment on $S K-N-A S, S K-N-B E(2)-C, S K-N-D Z, S K-N-F I$ and $S K-N-S H$ cells. Single treatments of all NB lines with
0.1-40 $\mu \mathrm{M}$ cisplatin, 0.001-1 $\mu \mathrm{M}$ vincristine, and 0.1-5 $\mu \mathrm{M}$ doxorubicin was followed for $72 \mathrm{~h}$ by WST-1 assays, and the data are presented in Fig. 4 and Table I. All NB lines responded to treatment in a dose-dependent manner, although their sensitivity varied, but was not associated with their sensitivity to the PI3K and FGFR inhibitors, as demonstrated by IC50 values 

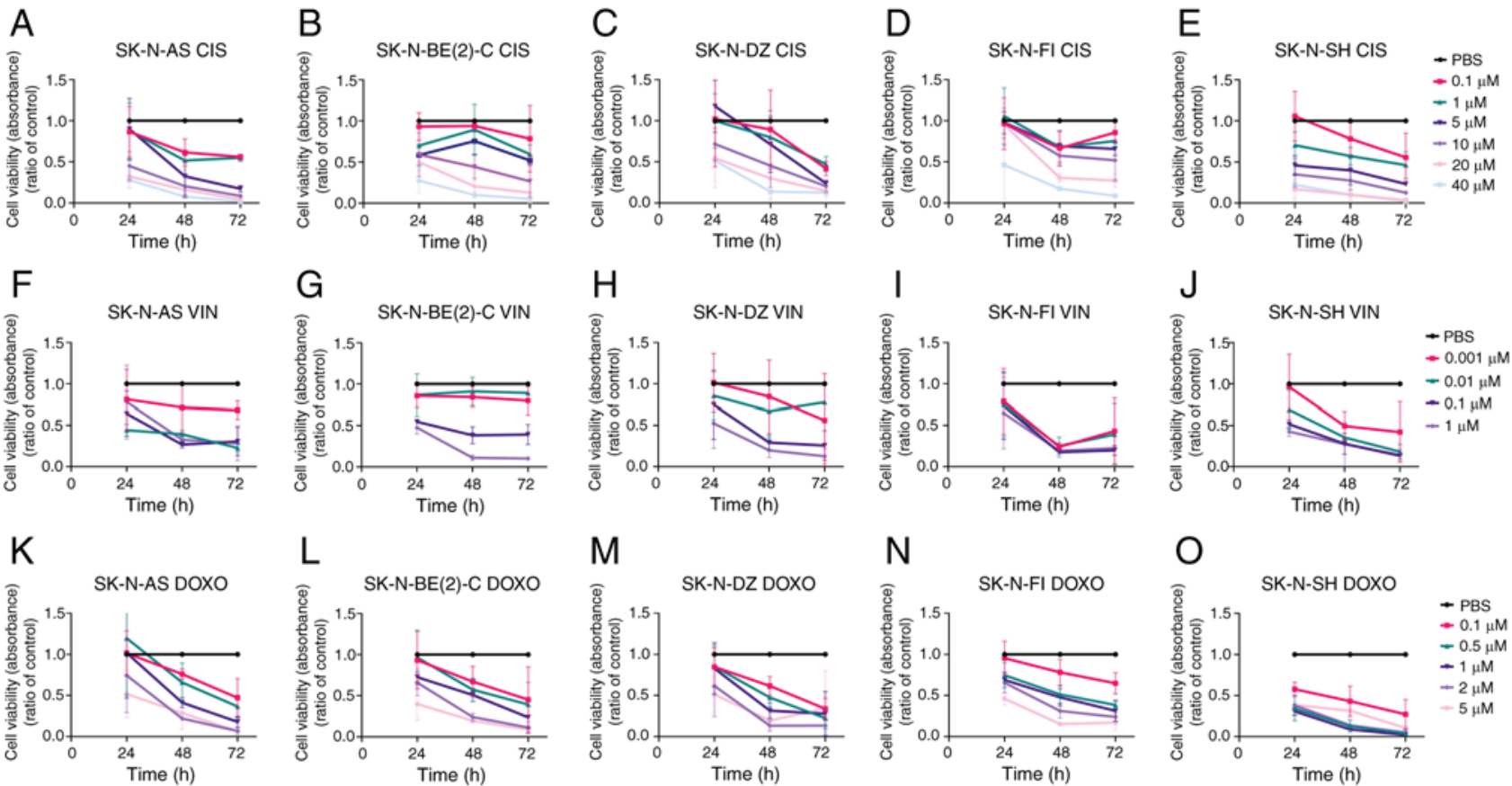

Figure 4. WST-1 viability assays on SK-N-AS, SK-N-BE(2)-C, SK-N-DZ, SK-N-FI and SK-N-SH cell lines upon treatment with cisplatin, vincristine and doxorubicin. WST-1 viability assay measured the absorbance following treatment for 24, 48 and $72 \mathrm{~h}$ of (A, F and K) SK-N-AS, (B, G and L) SK-N-BE(2)-C, (C, H and M) SK-N-DZ, (D, I and N) SK-N-FI, and (E, J and O) SK-N-SH cells treated with (A-E) cisplatin, (F-J) vincristine and (K-O) doxorubicin. The graphs represent 3 experimental runs per cell line and results are presented as the means \pm standard deviation. CIS, cisplatin; VIN, vincristine; DOXO, doxorubicin.

of the different NB lines to the different drugs and inhibitors (Table I). Nevertheless, the SK-N-SH cell line seemed to be sensitive to both the inhibitors and cytostatic drugs (Table I). The effects of single cytostatic drug treatments were further analyzed by proliferation, cytotoxicity and apoptosis assays (please see below).

Cisplatin. Concentration-dependent drug responses to cisplatin were observed for all NB cell lines. At low concentrations, the majority of the lines were resistant early on following treatment; however, after $72 \mathrm{~h}$, all exhibited a significantly decreased absorbance compared to PBS for all cisplatin concentrations $(0.1-40 \mu \mathrm{M})$, with the exception of the SK-N-BE(2)-C, SK-N-FI and SK-N-SH cells treated with the $0.1 \mu \mathrm{M}$ concentration (for all remaining, at least $\mathrm{P}<0.05$ ) Fig. 4A-E.

Vincristine. Concentration-dependent drug responses to vincristine were observed for all NB cell lines, and all presented significantly decreased absorbance compared to PBS at 48 and $72 \mathrm{~h}$ with the two highest concentrations $(0.1$ and $1.0 \mu \mathrm{M})$ (for all, at least $\mathrm{P}<0.05$ ) (Fig. 4F-J).

Doxorubicin. Concentration-dependent responses to doxorubicin were also observed for all NB lines. The SK-N-SH cells exhibited a decreased absorbance compared to PBS at all time points and concentrations (at least $\mathrm{P}<0.01$ ), and all the other NB lines were also sensitive to doxorubicin (for most, at least $\mathrm{P}<0.05$ ) (Fig. 4K-O).

To conclude, all NB cell lines exhibited concentration-dependent responses to the cytotoxic drugs and these varied depending on the cell line and drug used; however, this was not associated with their sensitivity to the FGFR and PI3K inhibitors (Table I). SK-N-SH was consistently the most chemo-sensitive cell line.

Effects of cisplatin, vincristine and doxorubicin in combination with the PI3K and FGFR inhibitors, BYL719 and $J N J-42756493$, on the $S K-N-B E(2)-C$ and $S K-N-S H$ cells. Given the positive combined effects with PI3K and FGFR inhibitors, the possible combined effects of the inhibitors with cytostatic drugs used clinically (please see above) were then examined. To this end, the NB lines, SK-N-BE(2)-C and SK-N-SH, were selected, the former with a MYCN amplification, was established from a relapsed, previously treated patient, and is regarded as relatively chemo-resistant, while the latter with wild-type 553 was sensitive to the majority of inhibitors and drugs tested. The SK-N-BE(2)-C and SK-N-SH cells were exposed to either BYL719 $(0.25-10 \mu \mathrm{M})$ or JNJ-42756493 (0.001-1.0 $\mu \mathrm{M})$ combined with cisplatin $(1-10 \mu \mathrm{M})$, vincristine (0.001-0.1 $\mu \mathrm{M})$, or doxorubicin (0.1-1 $\mu \mathrm{M})$ (Fig. 5).

The calculations of the combinational effect analysis and according to the dose-effect-based median-effect-principle after $24 \mathrm{~h}$ are presented in Fig. 6. In contrast to the predominantly positive combinational effects combining PI3K and FGFR inhibitors (Fig. 3), the combination of the inhibitors with the cytostatic drugs, resulted in more diverse effects, including neutral, positive and adverse effects (Figs. 5 and 6).

PI3K inhibitor, BYL719, in combination with cisplatin, vincristine or doxorubicin

$S K-N-B E(2)-C$ cells. Single effects for comparison to the combinational effects of cisplatin, vincristine, or doxorubicin with BYL719 evaluated by WST-1 assays of the SK-N-BE(2)-C 
A

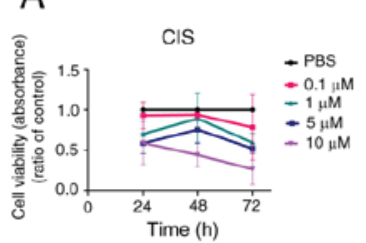

BYL

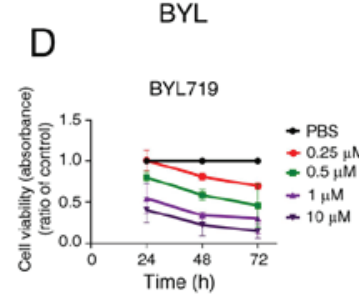

JNJ

$\mathrm{H}$

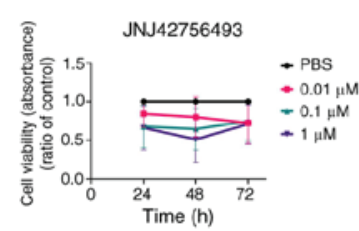

SK-N-SH

\section{E}

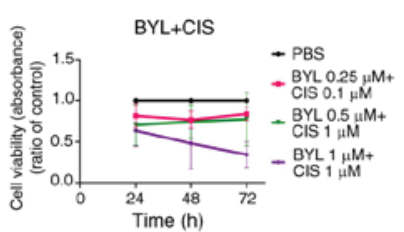

I

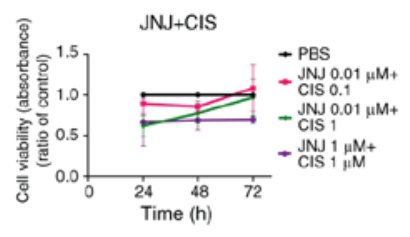

L

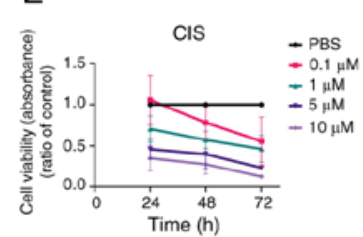

BYL

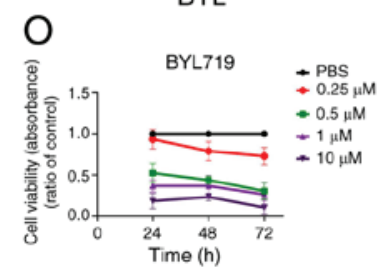

$\mathrm{P}$

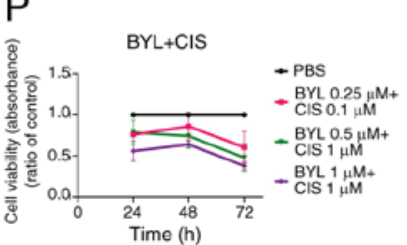

S

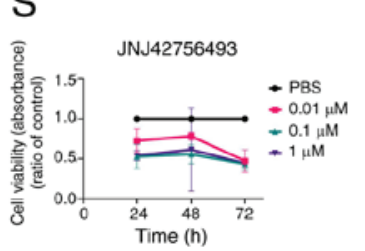

T

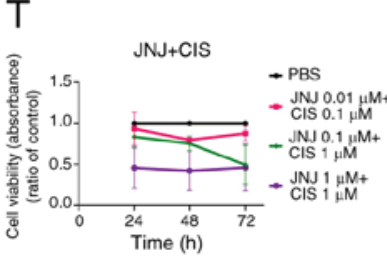

$\mathrm{B}$

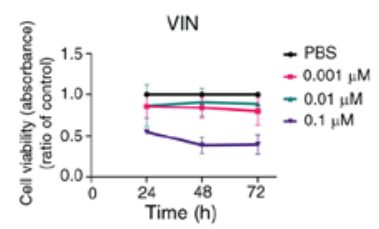

F

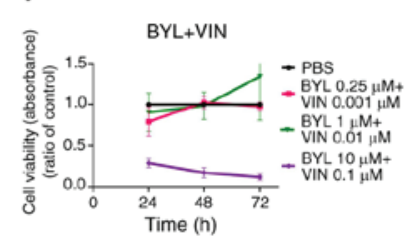

JNJ+CYTOSTATICS

J

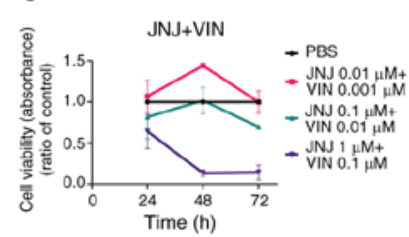

CYTOSTATICS

M

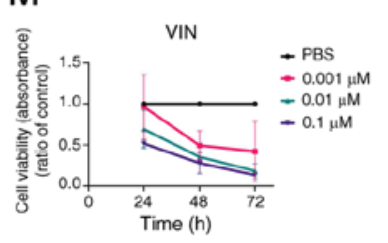

BYL+CYTOSTATICS

Q

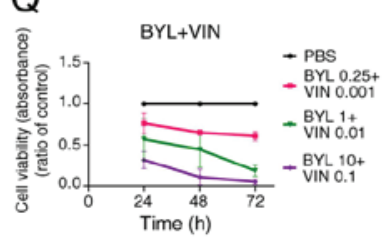

JNJ+CYTOSTATICS
U

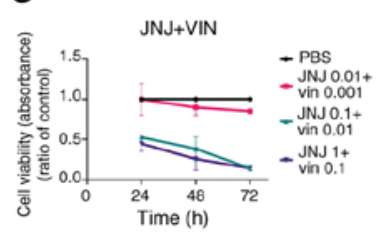

C

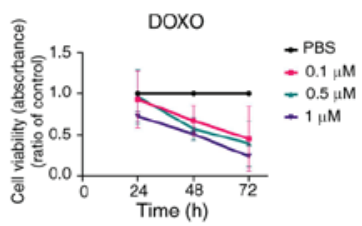

G

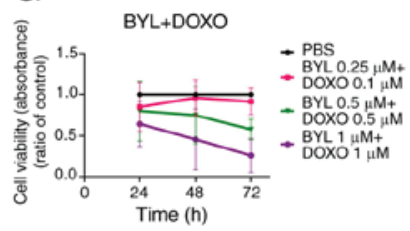

$\mathrm{K}$

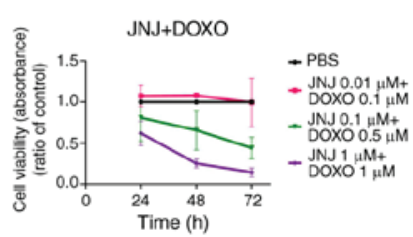

$\mathrm{N}$

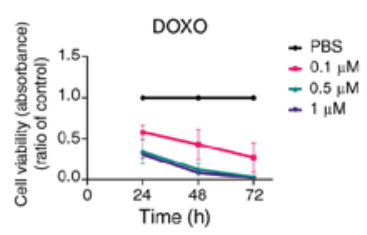

$\mathrm{R}$

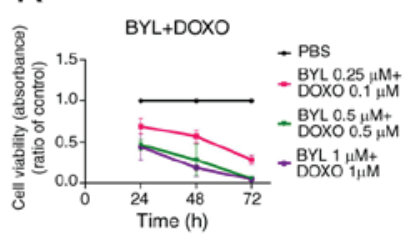

V

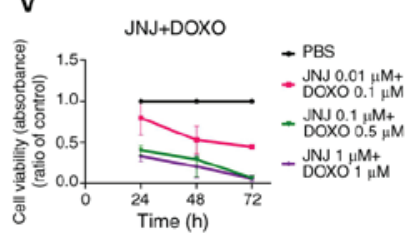

Figure 5. WST-1 viability assays on SK-N-BE(2)-C and SK-N-SH cell lines upon treatment with BYL719 or JNJ-42756493 with cisplatin, vincristine and doxorubicin. Viability measured as absorbance, 2448 and $72 \mathrm{~h}$ following treatment with cisplatin, vincristine or doxorubicin alone of (A-C) SK-N-BE(2)-C and (L-N) SK-N-SH cells, or (D) BYL719 treatment of SK-N-BE(2)-C cells and (O) treatment of SK-N-SH cells with BYL719, or (H) treatment of SK-N-BE(2)-C cells with JNJ-42756493, and (S) treatment of SK-N-SH cells with JNJ-42756493. Combined effect on the viability of (E) SK-NS-BE(2)-C and (P) SK-N-SH cells of BYL719 together with cisplatin. Combined effect on the viability of (F) SK-NS-BE(2)-C and (Q) SK-N-SH cells of BYL719 with vincristine. Combined effect on the viability of (G) SK-NS-BE(2)-C and (R) SK-N-SH cells of BYL719 and doxorubicin. Combined effect on the viability of (I) SK-N-BE(2)-C and (T) SK-N-SH cells of JNJ-42756493 together with cisplatin. Combined effect on the viability of (J) SK-NS-BE(2)-C and (U) SK-N-SH cells of JNJ-42756493 and vincristine. Combined effect on the viability of (K) SK-N-BE(2)-C and (V) SK-N-SH cells of JNJ-42756493 and doxorubicin. BYL, BYL719; JNJ, JNJ-42756493; CIS, cisplatin; VIN, vincristine; DOXO, doxorubicin.

cells are shown in Fig. $5 \mathrm{~A}-\mathrm{G}$ (for all at least $\mathrm{P}<0.05)$. The combination of BYL719 with cisplatin, vincristine or doxorubicin yielded dose-dependent responses, with diverse changes in absorbance compared to PBS, exhibiting additive, neutral or adversary effects of the drug combinations compared to the single-drug exposures (Fig. 5E-G). As shown in Fig. 6A and C, 

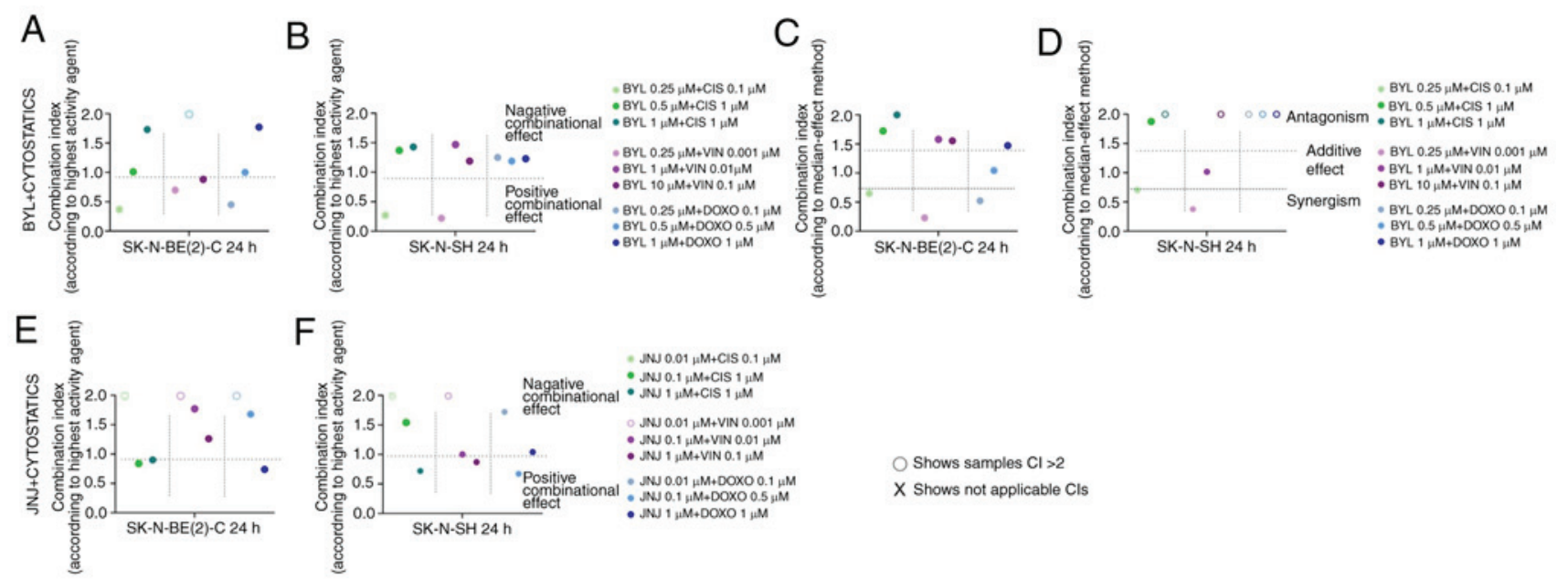

Figure 6. Combinational effects of the PI3K inhibitor, BYL719, and FGFR inhibitor, JNJ-42756493, with cisplatin, vincristine and doxorubicin on SK-N-BE(2)-C and SK-N-SH cell lines. CIs were obtained by the highest single agent approach following treatment of (A and E) SK-N-BE(2)-C and (B and F) SK-N-SH cells with (A and B) BYL719 or (E and F) JNJ-42756493 and cytostatic drugs. CIs were calculated also only for BYL719 and cytostatic drugs by using the median effect method for (C) SK-N-BE(2)-C and (D) SK-N-SH cells, since for JNJ-42756493 $\mathrm{r}<0.85$ in median method, so the analysis could not proceed. CIs were calculated from the mean of 3 experiments, analyzed by WST 1. o denotes CI >2, which shows a negative combination effect. CI, combination index; BYL, BYL719; JNJ, JNJ-42756493; CIS, cisplatin; VIN, vincristine; DOXO, doxorubicin.

some positive combinational effects $(\mathrm{CI}<1)$ e.g., synergistic effects for the SK-N-BE(2)-C cells, with $0.25 \mu \mathrm{M}$ BYL719 and $0.1 \mu \mathrm{M}$ cisplatin, $0.25 \mu \mathrm{M}$ BYL719 and $0.001 \mu \mathrm{M}$ vincristine, and $0.25 \mu \mathrm{M}$ BYL719 and $0.1 \mu \mathrm{M}$ doxorubicin were observed at $24 \mathrm{~h}$ following treatment, while the remaining data indicated neutral or adverse outcomes.

$S K$ - $N$-SH cells. Single effects for comparison to the combinational effects of cisplatin, vincristine, doxorubicin and BYL719 on the viability of SK-N-SH cells are shown in Fig. 5L-R. The majority of the combinations led to significant decreases in absorbance at all time points compared to PBS (for all at least $\mathrm{P}<0.05$ ) (Fig. 5P-R). Positive combinatory effects were however, not common; in fact, neutral or adverse outcomes were more frequent or equally present. This was confirmed by the calculation of the combinational effects and dose-effect-based median-effect-principle $24 \mathrm{~h}$ following treatment, as shown in Fig. 6B and D. Herein, $0.25 \mu$ M BYL719 and $0.1 \mu \mathrm{M}$ cisplatin, and $0.25 \mu \mathrm{M}$ BYL719 and $0.001 \mu \mathrm{M}$ vincristine exhibited synergy, while the remaining combinations led to either neutral or adverse consequences. For BYL719 and doxorubicin, the dose-effect-based median-effect-principle could not be calculated.

To conclude, the combinations of BYL719 with cisplatin, vincristine and doxorubicin used on the SK-N-BE(2)-C and SK-N-SH cells resulted in variable effects with both positive, neutral and adverse combinatory effects (Figs. 5 and 6). It was not possible to consistently state that any combination was the optimal for any of the cell lines. Notably, however, the lowest BYL719-cisplatin and lowest BYL719-vincristine combinations exerted a synergistic effect for both SK-N-BE(2)-C and SK-N-SH cells.

FGFR inhibitor, JNJ-42756493, in combination with cisplatin, vincristine and doxorubicin

$S K-N-B E(2)-C$ cells. The single and combined effects of cisplatin, vincristine, doxorubicin with JNJ-42756493 on the viability of SK-N-BE(2)-C cells are shown in Fig. 5A-C, H and I-K, respectively. No clear-cut enhanced sensitivity was observed upon the combination of JNJ-42756493 with the cytostatic drugs. This was confirmed by the calculation of the combinational effects after $24 \mathrm{~h}$, where only some rare positive effects were observed (Fig. 6E), while the dose-effect-based median-effect-principle could not be calculated. Positive effects were observed after $24 \mathrm{~h}$ with the 0.01 and $0.1 \mu \mathrm{M}$, and 0.1 and $1 \mu \mathrm{M} \mathrm{JNJ}-42756493$ and cisplatin combinations, and the $1 \mu \mathrm{M} \mathrm{JNJ}-42756493$ and $1 \mu \mathrm{M}$ doxorubicin combination, while remaining outcomes tended to be neutral or adverse (Fig. 6E).

$S K-N$-SH cells. The single and combinational effects of cisplatin, vincristine, doxorubicin and JNJ-42756493 on the viability of SK-N-SH cells are shown in Fig. 5L-N, S and T-V, respectively. All combinations significantly decreased viability compared to PBS at $72 \mathrm{~h}$ following treatment except for the $0.01 \mathrm{JNJ}-43756493$ and $0.1 \mu \mathrm{M}$ cisplatin combination (for all, $\mathrm{P}<0.05$ at least), although positive effects were not dominant (Figs. 5T-V, and 6F). Positive combinational effects were observed after $24 \mathrm{~h}$ for the $1 \mu \mathrm{M} \mathrm{JNJ}-42756493$ and $\mu \mathrm{M}$ cisplatin combination, the $0.1 \mu \mathrm{M} \mathrm{JNJ}-42756493$ and $1 \mu \mathrm{M}$ vincristine combination, and the $0.1 \mu \mathrm{M} \mathrm{JNJ}-42756493$ and $0.5 \mu \mathrm{M}$ doxorubicin combination, while the remaining outcomes were neutral or adverse (Fig. 6F). Dose-effect-based median-effect-principle could not be calculated

To sum up, the combined effects of JNJ-42756493 with cisplatin, vincristine and doxorubicin on the SK-N-BE(2)-C and SK-N-SH cells yielded variable outcomes with positive, neutral and adverse effects (Figs. 5 and 6). It was not possible to state a specific combination as the most effective for inhibiting viability in any of the cell lines (Fig. 6).

Proliferation, apoptosis and cytotoxicity following single and combined treatment of $S K-N-B E(2)-C$ and $S K-N-S H$ cells with PI3K and FGFR inhibitors. After conducting WST-1 
A

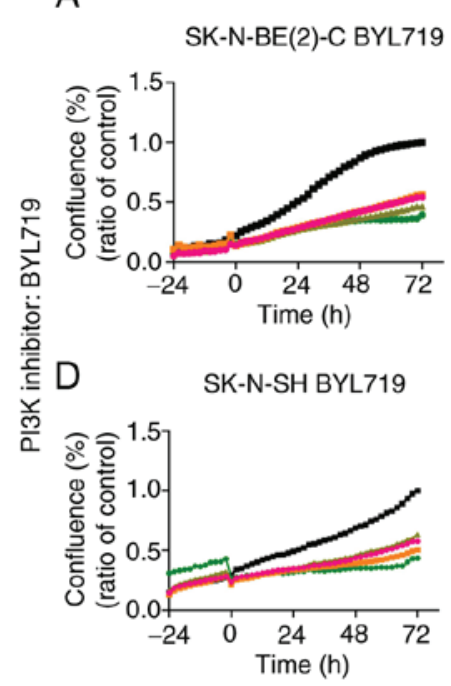

G

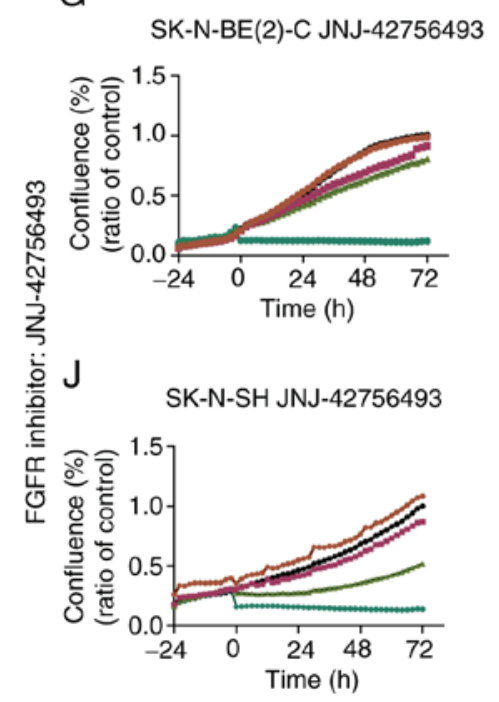

B

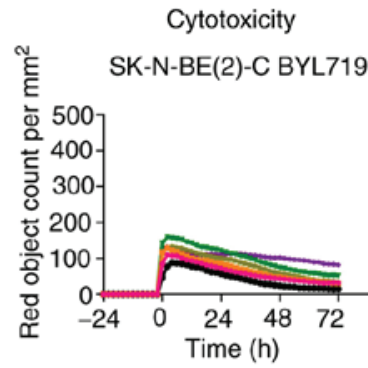

E

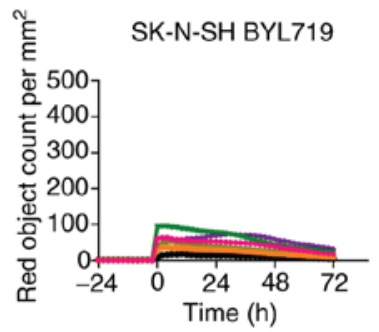

$\mathrm{H}$

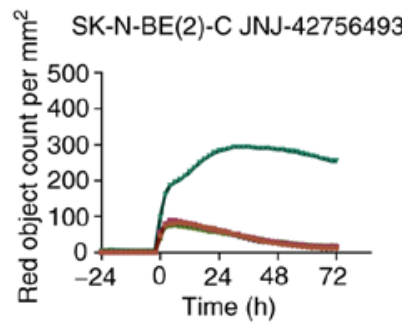

$\mathrm{K}$

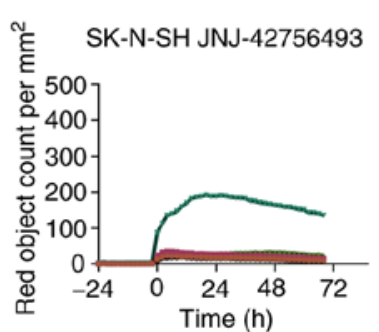

C

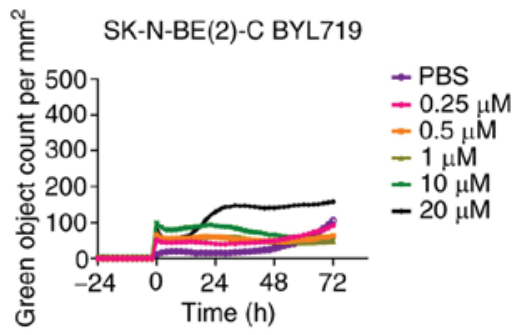

F

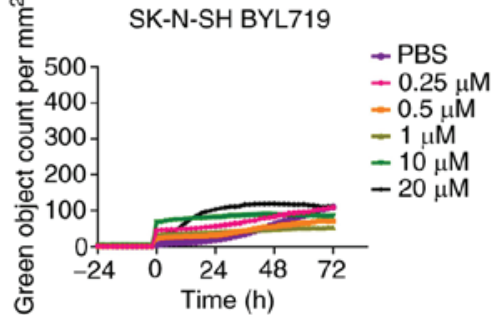

I

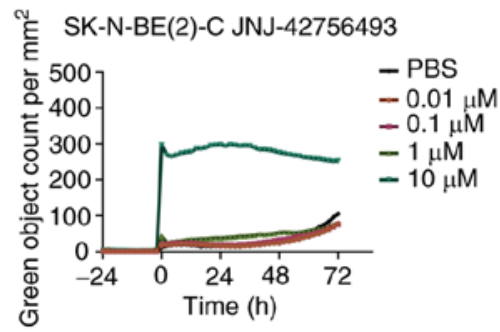

L

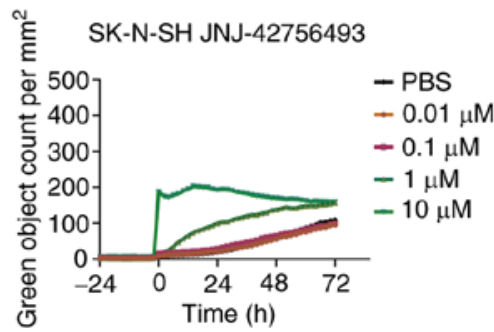

Figure 7. Proliferation, cytotoxicity and apoptosis on SK-N-BE(2)-C and SK-N-SH cell lines upon treatment with BYL719 and JNJ-42756493. Proliferation, cytotoxicity and apoptosis response to BYL719 (PI3K inhibitor) for (A-C) SK-N-BE(2)-C cells and for (D-F) SK-N-SH cells. Proliferation, cytotoxicity and apoptosis response to JNJ-42756493 (FGFR inhibitor) for (G-I) SK-N-BE(2)-C cells and for (J-L) SK-N-SH cells. The graphs represent 3 experimental runs per cell line.

assays (as described above), single and combined inhibitor treatments were performed and proliferation, apoptosis and cytotoxicity were examined using the IncuCyte S3 Live-Cell Analysis System on the BYL719 and JNJ-42756493, and SK-N-BE(2)-C and SK-N-SH cells. This analysis system could more specifically reflect upon the joint data reflected in the WST-1 assay, by distinguishing proliferation, cytotoxicity in a more specific manner.

Single treatments of $S K-N-B E(2)-C$ and $S K-N$-SH cells with $P I 3 K$ and FGFR inhibitors

BYL719 and JNJ-42756493. The proliferation, apoptosis and cytotoxicity of the SK-N-BE(2)-C and SK-N-SH cells were observed $72 \mathrm{~h}$ following treatment with BYL719 $(0.25-20 \mu \mathrm{M})$ and JNJ-42756493 (0.01-10 $\mu \mathrm{M})$. Both cell lines exhibited dose-dependent decreases in proliferation to both drugs, and with the drug concentrations used, the highest JNJ-42756493 concentration induced very marked cytotoxicity and apoptosis, with the SK-N-BE(2)-C cells tending to present higher cytotoxicity and apoptosis than the SK-N-SH cells (Fig. 7). Images of the effects on proliferation are depicted in Fig. S1.

BEZ235 and AZD4547. Both BEZ235 and AZD4547 have previously been reported to induce dose-dependent proliferation inhibition, and, in line with the present study, the FGFR inhibitor, AZD4547, induced more pronounced effects than the PI3K inhibitor on cytotoxicity and the apoptosis of SK-N-BE(2)-C and SK-N-SH cells (14).

Combined treatments of $S K-N-B E(2)-C$ and $S K-N-S H$ cells with PI3K and FGFR inhibitor. Effects on proliferation, cytotoxicity and apoptosis upon combined treatment with PI3K and FGFR inhibitors are presented for the SK-N-BE(2)-C and SK-N-SH cells.

BYL719 and JNJ-42756493. The proliferation, apoptosis and cytotoxicity of the SK-N-BE(2)-C and SK-N-SH cells were observed for $72 \mathrm{~h}$ following treatment with combinations of 
A

Proliferation
SK-N-BE(2)-C BYL-JNJ

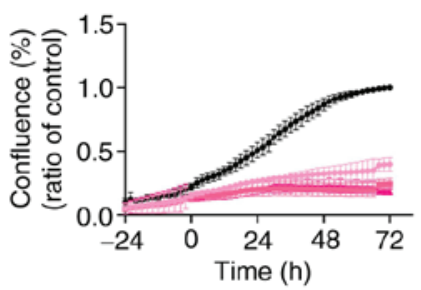

D

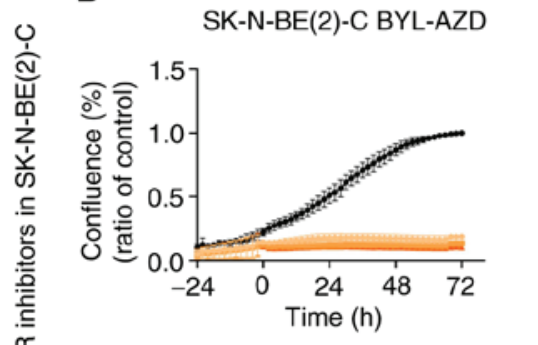

$\mathrm{G}$

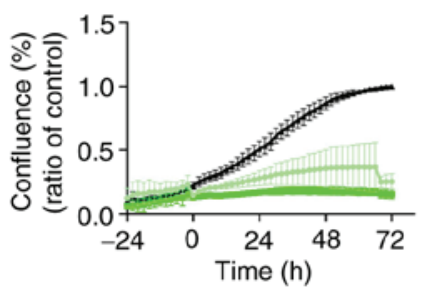

J

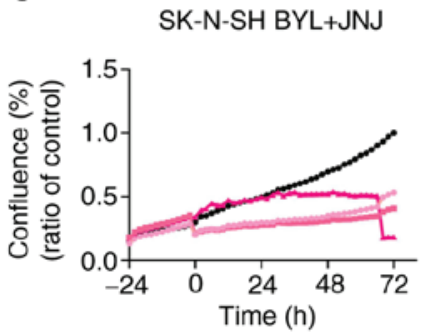

$\mathrm{M}$

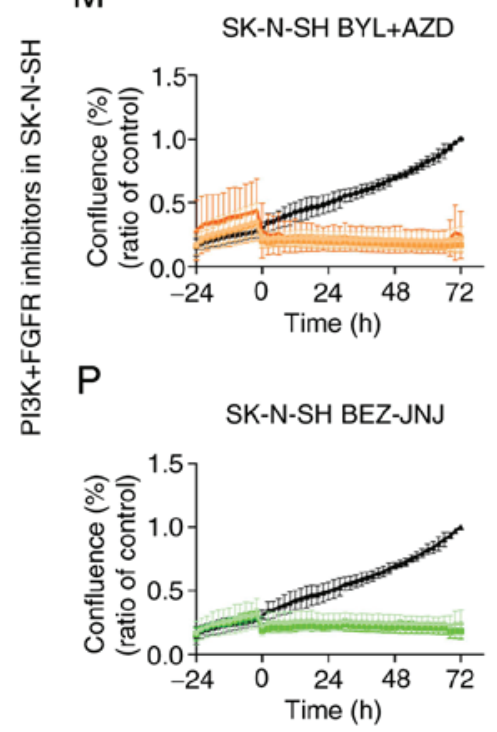

B Cytotoxicity
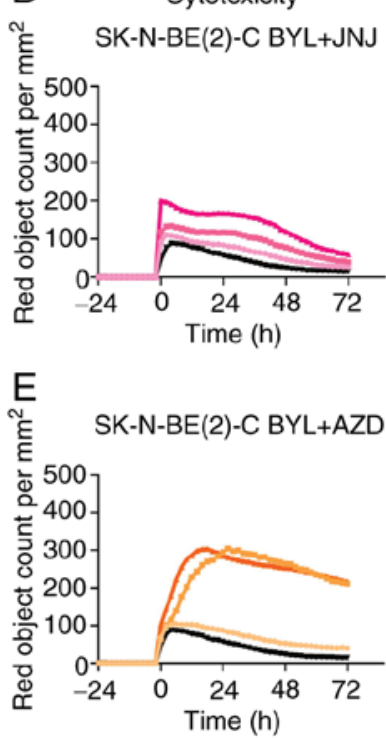

$\mathrm{H}$
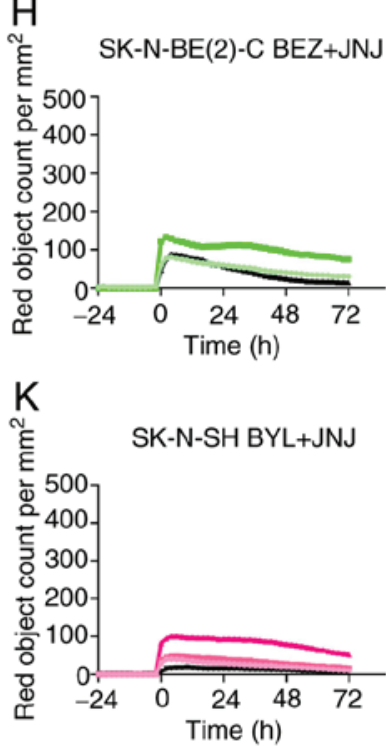

N
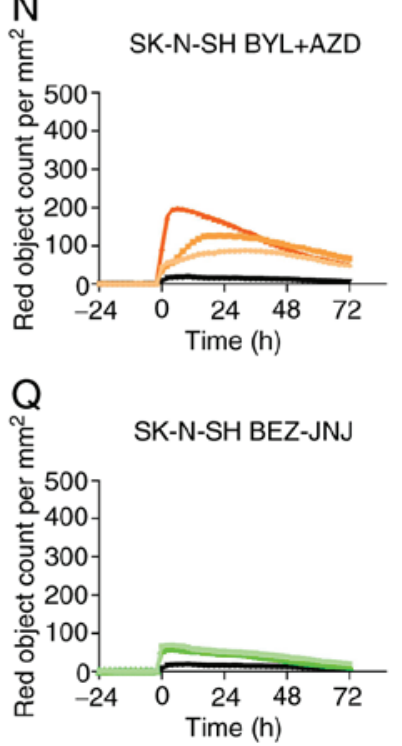

C ข

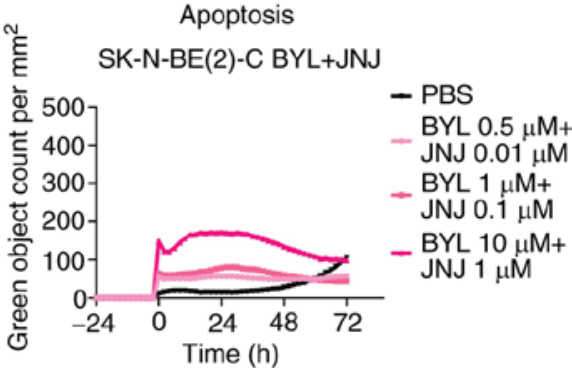

$\mathrm{F}$
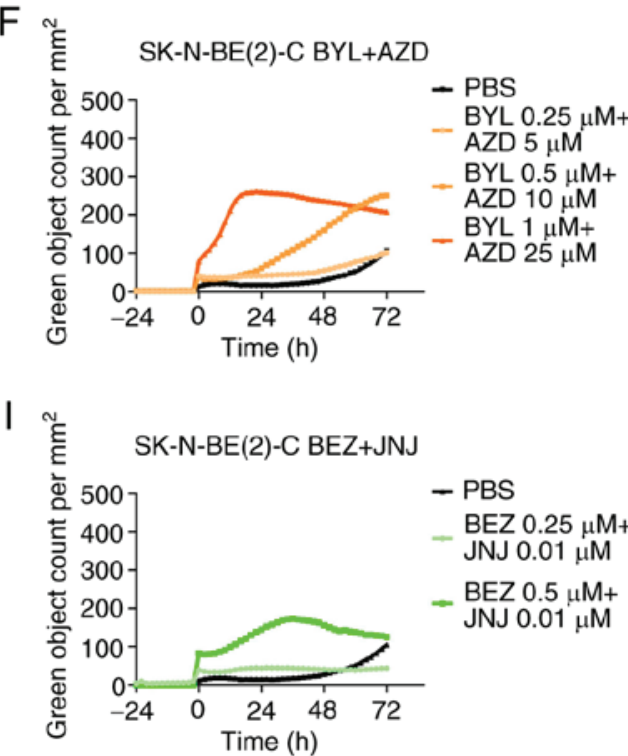

$\mathrm{L}$
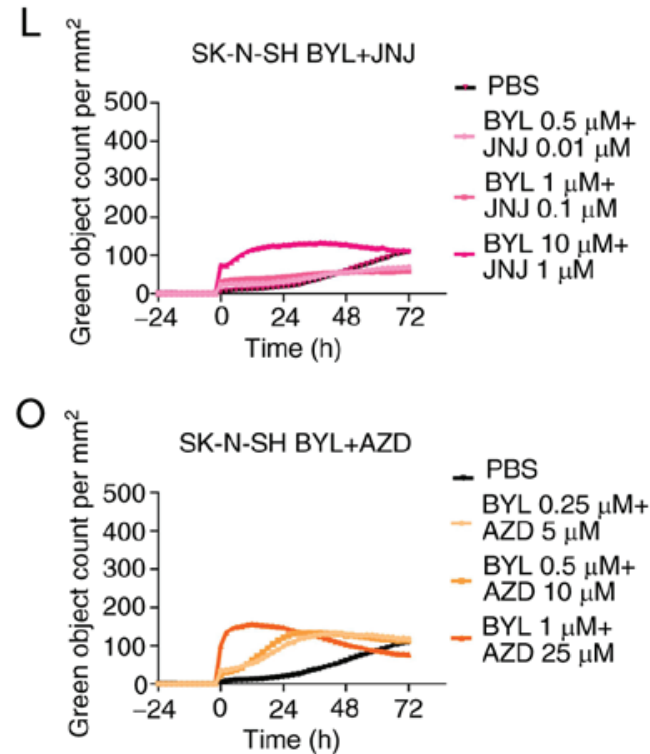

R

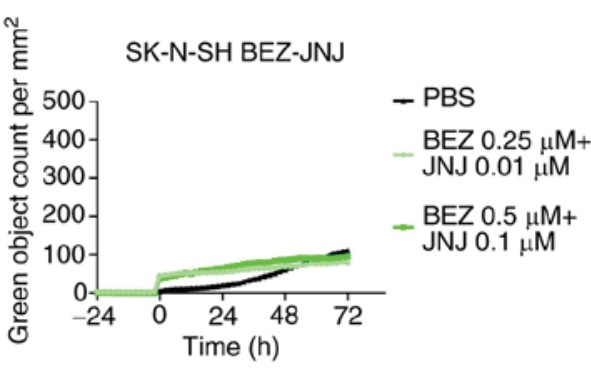

Figure 8. Proliferation, cytotoxicity and apoptosis on SK-N-BE(2)-C and SK-N-SH cell lines upon treatment with combinations of PI3K (BYL719 and BEZ235) and FGFR (JNJ-42756493 and AZD4547) inhibitors. Combined effects on proliferation, cytotoxicity and apoptosis of PI3K inhibitors (BYL719 and BEZ235) with FGFR inhibitors (JNJ-42756493 and AZD4547) on (A-I) SK-N-BE(2)-C cells and (J-R) SK-N-SH cells. The graphs represent 3 experimental runs per cell line. BYL, BYL719; JNJ, JNJ-42756493; BEZ, BEZ235; AZD, AZD4547. 


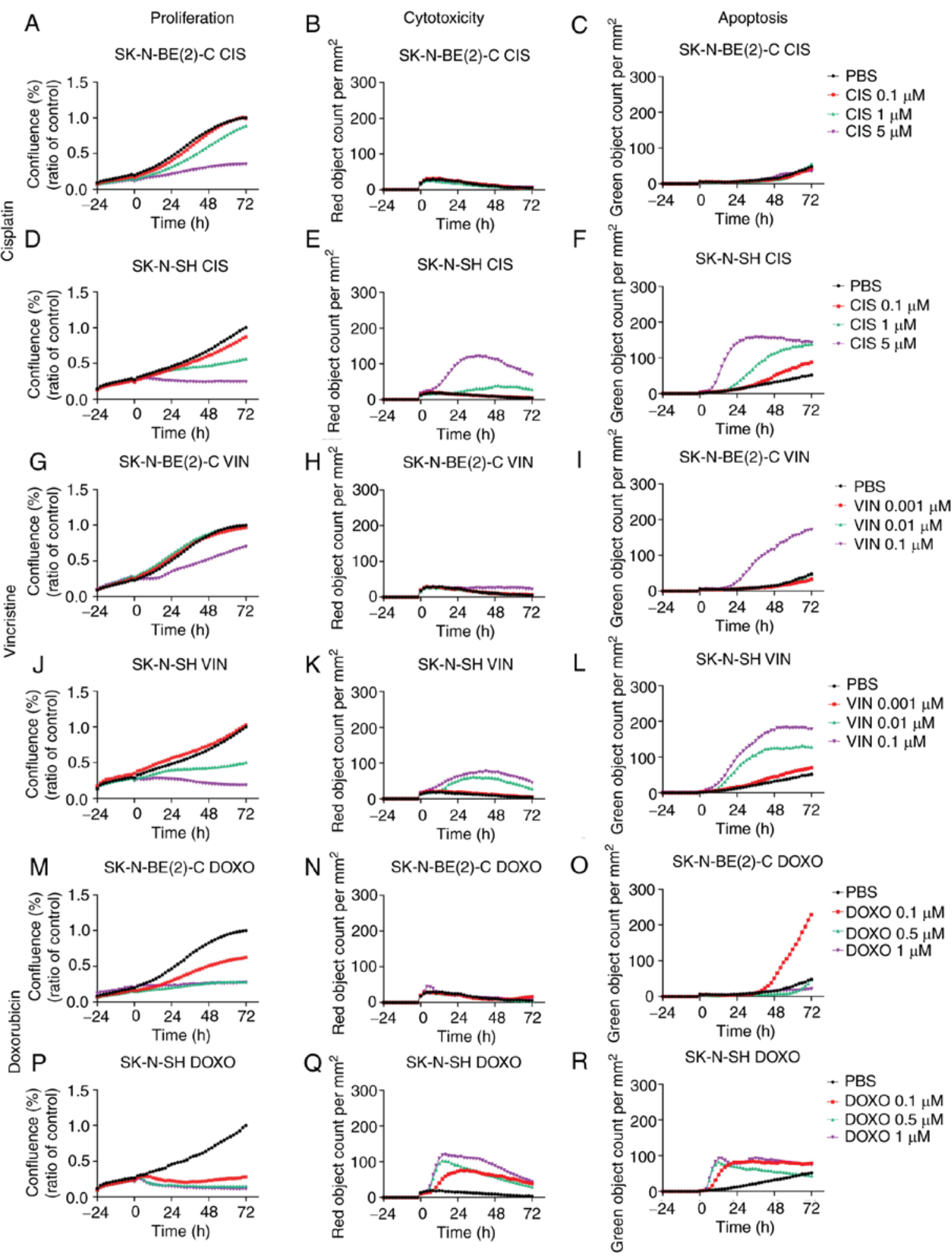

Figure 9. Proliferation, cytotoxicity and apoptosis on SK-N-BE(2)-C and SK-N-SH cell lines upon treatment with cisplatin, vincristine or doxorubicin. Effects on proliferation, cytotoxicity and apoptosis of cisplatin, vincristine and doxorubicin, respectively on SK-N-BE(2)-C cells (A-C, G-I and M-O, respectively), and on SK-N-SH cells (D-F, J-L and P-R, respectively). The graphs represent 3 experimental runs per cell line. CIS, cisplatin; VIN, vincristine; DOXO, doxorubicin.

BYL719 $(0.5-10 \mu \mathrm{M})$ and JNJ-42756493 (0.01-1 $\mu \mathrm{M})$. Both cell lines exhibited a marked decrease in proliferation, while the effects on cytotoxicity and apoptosis were consistently more pronounced for the SK-N-BE(2)-C than for the SK-N-SH cells (Fig. 8A-C and J-L, respectively), with images on proliferation presented in Fig. S1.
BYL719 and AZD4547, as well as BEZ235 and JNJ-42756493. The FDA-approved inhibitors were also combined with the previously tested inhibitors at the drug concentrations indicated above. For both the SK-N-BE(2)-C and SK-N-SH cells, an enhanced decrease in proliferation was noted at all time points with all concentrations (Fig. 8D, G and M, and P, 
respectively). For all combinations, cytotoxicity and apoptosis were consistently observed more readily for the SK-N-BE(2)-C cells when compared to the SK-N-SH cells (Fig. 8).

To conclude, in both the SK-N-BE(2)-C and SK-N-SH cells, the combined use of PI3K and FGFR inhibitors resulted in an enhanced inhibition of proliferation, in parallel with data obtained from the WST-1 assays. Effects on cytotoxicity and apoptosis were consistently observed more readily in the SK-N-BE(2)-C cells than in the SK-N-SH cells.

Proliferation, apoptosis and cytotoxicity following treatment of $S K-N-B E(2)-C$ and $S K-N-S H$ cells with single cytostatic drugs, or cytostatic drugs combined with PI3K and FGFR inhibitors. After conducting WST-1 assays, with the single cytostatic drugs, cisplatin, vincristine and doxorubicin, or in combination with BYL719 and JNJ-42756493, their effects on proliferation, apoptosis and cytotoxicity in the SK-N-BE(2)-C and SK-N-SH cells were observed (Fig. 9). Images of proliferation are presented in Fig. S2.

Single treatments of $S K-N-B E(2)-C$ and $S K-N-S H$ cells with cytostatic drugs

Cisplatin. The effects on proliferation, cytotoxicity and apoptosis following treatment with $0.1-5 \mu \mathrm{M}$ cisplatin were observed for $72 \mathrm{~h}$ for the SK-N-BE(2)-C and SK-N-SH cells and the results are depicted in Fig. 9A-C and D-F, respectively. Dose-dependent effects on proliferation were observed for both cell lines, while the effects on cytotoxicity and apoptosis were minimal for the SK-N-BE(2)-C cells as compared to those for the SK-N-SH cells.

Vincristine. The effects on proliferation, cytotoxicity and apoptosis following treatment with $0.001-0.1 \mu \mathrm{M}$ vincristine were observed for $72 \mathrm{~h}$ for the SK-N-BE(2)-C and SK-N-SH cells and are depicted in Fig. 9G-I and J-L, respectively. Dose-dependent effects on proliferation were observed for both lines, with the SK-N-SH cells being more sensitive to the lower drug concentrations than the SK-N-BE(2)-C cells, in concordance with the higher cytotoxicity and apoptotic levels of the SK-N-SH cells compared to the SK-N-BE(2)-C cells.

Doxorubicin. The effects on proliferation, cytotoxicity and apoptosis following treatment with $0.1-1 \mu \mathrm{M}$ doxorubicin were observed for $72 \mathrm{~h}$ for the SK-N-BE(2)-C and SK-N-SH cells and the results are depicted in Fig. 9M-O and P-R, respectively. Dose-dependent effects on proliferation were observed for both cell lines, with the SK-N-SH cells being more sensitive to the lower drug concentrations than the SK-N-BE(2)-C cells, in concordance also with the higher cytotoxicity and apoptotic levels of the SK-N-SH cells as compared to the SK-N-BE(2)-C cells.

To conclude, single cisplatin, vincristine and doxorubicin treatments, although not including the highest concentrations present in the WST-1 assays, exerted dose-dependent effects on both the SK-N-BE(2)-C and SK-N-SH cells. The SK-N-SH cells, being the more chemo-sensitive line as compared to the SK-N-BE(2)-C cells, was consistently more sensitive to the lower drug concentrations than the SK-N-BE(2)-C cells, with regard to the inhibition of proliferation and effects on cytotoxicity and apoptosis.
Combined treatment of $S K-N-B E(2)-C$ and $S K-N-S H$ cells with PI3K or FGFR inhibitor with cytostatic drugs. The effects on proliferation, cytotoxicity and apoptosis with cisplatin $(0.1-1 \mu \mathrm{M})$, vincristine $(0.001-0.1 \mu \mathrm{M})$, or doxorubicin $(0.1-1 \mu \mathrm{M})$, combined with BYL719, $(0.25-10 \mu \mathrm{M})$ or JNJ-42756493 (0.01-10 $\mu \mathrm{M})$, i.e., excluding higher single concentrations, on the SK-N-BE(2)-C and SK-N-SH cells were observed for $72 \mathrm{~h}$ (data not shown). In the proliferation analysis, dose-dependent effects were obtained with all combinations, but with no clear-cut positive nor negative effects of the combinations, while the effects on cytotoxicity and apoptosis were moderate with the drug concentrations used.

To summarize, dose-dependent effects on proliferation, were observed upon combining BYL719 or JNJ-42756493 with either cisplatin, vincristine or doxorubicin; however, no clear-cut positive effects were acquired and modifications of the effects on cytotoxicity and apoptosis were moderate or minimal with the inhibitor and drug doses used.

\section{Discussion}

In the present study, the recently FDA-approved drugs, alpelisib (PI3K inhibitor) and erdafitinib (FGFR inhibitor), were shown to exert dose-dependent effects with decreased viability and proliferation on the $5 \mathrm{NB}$ cell lines, SK-N-AS, SK-N-BE(2)-C, SK-N-DZ, SK-N-FI and SK-N-SH. Importantly, this was also the case for NB cell lines with specific high-risk mutations or MYCN amplification. Moreover, upon combination with the inhibitors, additive/synergistic effects were observed with a similar decrease in viability and proliferation using lower concentrations of the inhibitors.

The 5 NB cell lines were also shown to exhibit dose-dependent effects with a decreased viability and proliferation upon exposure to cisplatin, vincristine and doxorubicin, commonly used clinically, although e.g., the SK-N-BE(2)-C and SK-N-DZ cells with MYCN amplifications were relatively more resistant. Subsequently, the SK-N-BE(2)-C cells, with MYCN amplification, and presumed drug-resistant, and the SK-N-SH cells, with wild-type p53 and presumed drug-sensitive, were examined for their sensitivity to inhibitor drug combinations; however, when combining drugs and inhibitors, more complex effects were noted.

The results of the inhibitors, alpelisib and erdafitinib, were consistent with the effects on viability and proliferation, and the cytotoxicity of the previously tested inhibitors, BEZ235 (PI3K inhibitor) and AZD4547 (FGFR inhibitor), on NB and medulloblastoma (MB) cell lines $(14,20)$, suggesting on-target effects of alpelisib and erdafitinib. Hence, the FDA-approved PI3K and FGFR inhibitors may be of interest for future clinical evaluation in children with refractory or recurrent NB or MB.

The fact that all NB lines exhibited drug-dependent dose responses and decreases in viability and proliferation, to FDA-approved alpesilib (BYL719) and erdafitinib (JNJ-42756493), and that the effects were enhanced upon combining the two drugs was not unexpected, since they had responded similarly to analogous inhibitors, BEZ235 and AZD4547 (14). Upon combined treatments with BYL719 and JNJ-42756493, the SK-N-SH and SK-N-FI cells tended to be the most sensitive lines; however, with the majority inhibitor combinations, all NB lines seemed susceptible, with possibly 
the SK-N-AS cells being marginally more resistant. It is possible that that the sensitivity of the SK-N-SH cells is due to the fact that these cells have a normal p53 expression, while the relative resistance of the SK-N-BE(2)-C and SK-N-DZ cells may be due to the fact that these cells have MYCN amplifications (14). Thus far however, there is no specific explanation for the relative sensitivity of the SK-N-FI cells, and the relatively greater resistance of the SK-N-AS cells to the above-mentioned inhibitors, since both do not have MYCN amplification and have mutated p53. Additional, studies would be required to elucidate the influence of the different inhibitors on specific mechanisms of action in the signaling pathways of the different NB cell lines.

Notably, the data described above emphasize the fact that NB cell lines, despite their heterogeneity and without having FGFR or PI3K mutations, can be sensitive to PI3K and FGFR inhibitors. This has also been supported by previous studies by others and us, where different tumors and tumor lines have been reported to be sensitive to PI3K and FGFR inhibitors, despite not having PI3K and or FGFR mutations or chromosomal rearrangements $(14,20,25-33)$. More specifically, in some reports, it was shown that having mutations conferred enhanced drug vulnerability, while this was not at all the case in other studies $(32,33)$. Combinatorial studies showing an enhanced efficacy on the inhibition of viability and proliferation, when combining BYL719 and JNJ-42756493 also emphasized consistency with previous data, indicating that PI3K and FGFR inhibitors can be combined and show synergistic activity $(14,20,25)$.

Moreover, apart from increasing the antitumor efficacy, synergistic combinations might also allow the use of lower concentrations of the single drugs, thereby possibly reducing side-effects. Eventually, targeting NB with 2 different mechanisms might reduce the risk of the development of resistance. That JNJ-42756493, at the concentrations used, exerted more prominent effects on cytotoxicity and apoptosis compared to BYL719, was not unexpected either, and was in line with reports on AZD4547 (FGFR inhibitor) being superior to the included PI3K inhibitors with regard to inducing cytotoxicity and apoptosis $(11,14,20,25)$. Thus, collectively, our data of the present study showing synergy of the 2 FDA-approved inhibitors, BYL719 and JNJ-42756493, allowing for the use of lower concentrations of the drugs and avoiding resistance, suggest that they indeed could be of clinical interest for the treatment of refractory or recurrent NB.

The 5 NB cell lines were also tested for their sensitivity to single therapies with cisplatin, vincristine and doxorubicin. Notably, herein, the SK-N-AS and SK-N-SH were the most sensitive cell lines to cisplatin and vincristine, including the SK-N-FI cells for the latter, while the SK-N-BE(2)-C cells were generally more resistant. These findings were not entirely unexpected, since several reports have investigated the sensitivity of NB cell lines to cytostatic drugs and repeatedly found SK-N-BE(2)-C being relatively more chemo-resistant and SK-N-SH being more chemo-sensitive (34-36). This was also reflected by the generally more prominent effects on cytotoxicity and apoptosis the cytotoxic drugs had on the latter, as compared to the former (Fig. 9), while the opposite was observed for the inhibitors (Fig. 7). Nevertheless, of note, all NB cell lines tended to be relatively more sensitive to doxorubicin at the concentrations used in the present study; in addition, herein, the SK-N-SH cell line was the most sensitive, a finding which is consistent with a previous report (37).

Therefore, when investigating possible additive effects using canonical cytostatic drugs combined with BYL719 or JNJ-42756493, the 2 cell lines, SK-N-BE(2)-C, which had an MYCN amplification and were fairly chemo-resistant, and SK-N-SH, which was generally chemo-sensitive to most drugs, were selected for comparison. Herein, a more complex image was obtained, with synergistic, additive, neutral and adverse effects. Of note however, were the potentially synergistic combinations of $0.25 \mu \mathrm{M}$ BYL719 and $0.1 \mu \mathrm{M}$ cisplatin, $0.25 \mu \mathrm{M}$ BYL719 and $0.001 \mu \mathrm{M}$ vincristine for both cell lines, as well as $0.25 \mu \mathrm{M}$ BYL719 and $0.1 \mu \mathrm{M}$ doxorubicin for the SK-N-BE(2)-C cells (Fig. 6C and D). These data suggest that experimentally, synergistic effects with low concentrations of inhibitors and drugs, could be easier to disclose, particularly on cell lines that are more resistant to both inhibitors and drugs, than using higher concentrations and more sensitive cell lines.

To the best of our knowledge, in an experimental setting, potential synergism between alpesilib and erdafitinib and cisplatin, vincristine and doxorubicin has not been tested previously in NB, and there are only limited reports on other cell lines with some of the present combinations. One report on nasopharyngeal cancer cell lines, demonstrated neutral or very mild additive effects upon combining similar doses of BYL719 and cisplatin to those used herein (38). The fact that the present study obtained not only positive, but also neutral and adverse effects, could at least partially be explained by the fact that BYL719 tends to induce G0/G1 arrest, and thereby could have inhibited some of the cytotoxic effects that e.g., cisplatin has $(38,39)$. Other studies have explored combinations of alpesilib with olaparib and erdafitinib with check point inhibitors $(29,40)$. While there is an apparent plethora of possible combinations with other anti-neoplastic agents, the present study focused on combinations with established NB drugs as this might more directly lead to clinical translation.

There are some limitations to the present study. Although 5 NB cell lines were examined, additional cell lines could have been included. Nevertheless, these NB lines are representative of the ones commonly used by the scientific community (15-18,35). Furthermore, the present study mainly focused on the effects the inhibitors and the cytotoxic drugs on viability, cytotoxicity and apoptosis, using well-established methods, rather than a more detailed analysis of signaling pathways, which the authors also plan to pursue in the future. Nonetheless, importantly, the data indicate that drug-drug interactions of PI3K and FGFR inhibitors with cytotoxic drugs are of a complex nature, and can paradoxically result in either synergistic or antagonistic interaction. While broader concentration ranges, modified incubation times, and sequential drug exposure might shed further light on the determinants of the quality of drug-drug interactions with respect to their anti-tumor efficacy, the drug and inhibitor concentrations used herein adhere to commonly used standard conditions and therefore allow more direct comparisons $(19,21,32,38,39)$.

As mentioned above, further studies are required to elucidate the mechanistic details of how the tested drug combinations exert synergistic or antagonistic effects to provide a 
pre-clinical rationale of how to test these combinations clinically.

To conclude, the present study provides evidence that the combined use of the FDA-approved drugs, alpelisib and erdafitinib, enhances their individual efficacy on viability and proliferation of well-established NB-cell lines, indicating their use could be helpful for the treatment of refractory or recurrent NB. In addition, the present study indicates that the incorporation of alpelisib and erdafitinib into clinical chemotherapy regimens, will require careful consideration in order to obtain the best efficacy.

\section{Acknowledgements}

Not applicable.

\section{Funding}

The present study was supported by the Swedish Childhoods Cancer Fond (PR2017-0042, PR2017-0052), the Swedish Cancer Society (180440, 2017/658), the Stockholm Cancer Society (181053), the Swedish Cancer and Allergy Foundation (190), the Royal Swedish Academy of Sciences (2017-2018), the Stockholm City Council (20180037), Stiftelsen AnnaBrita o Bo Casters Minne (Lindhés Advokatbyrå) (LA2019-0080, LA2020-0012), Svenska Läkaresällskapets (SLS-934161), and Karolinska Institutet, Sweden (2018:0007).

\section{Availability of data and materials}

All data generated or analyzed during this study are included in this published article or are available from the corresponding author on reasonable request.

\section{Authors' contributions}

ONK and SH, performed the majority of the experiments, interpreted the data, calculated the statistics and contributed to the writing of the manuscript. ML collaborated with $\mathrm{ONK}$ and $\mathrm{SH}$ and performed some experiments, and ML contributed together with $\mathrm{ONK}$ and $\mathrm{SH}$ in preparing the graphs of the manuscript. CV and CP initiated the experiments and the interpretation of the initial experiments and contributed to the writing of the material and methods section, all under the supervision of ONK. MW assisted in the combinational analyses and in the final interpretation of the data. $\mathrm{NH}$ assisted in the analysis of the data and in their clinical interpretation. TD and ONK made substantial contributions to the conception and design of the study, the acquisition of data, analysis and interpretation of data, and were involved in drafting the manuscript and revising it critically for important intellectual content. TD also provided the sources for the performance of the experiments. TD and ONK provided the financial support for conducting the research. All authors critically read and approved the final manuscript.

\section{Ethics approval and consent to participate}

Not applicable.

\section{Patient consent for publication}

Not applicable.

\section{Competing interests}

The authors declare they have no competing interests.

\section{References}

1. Ward E, DeSantis C, Robbins A, Kohler B and Jemal A: Childhood and adolescent cancer statistics, 2014. CA Cancer J Clin 64: 83-103, 2014.

2. Park JR, Bagatell R, London WB, Maris JM, Cohn SL, Mattay KK and Hogarty M: COG Neuroblastoma Committee. Children's Oncology Group's 2013 blueprint for research: Neuroblastoma. Pediatr Blood Cancer 60: 985-993, 2013.

3. London WB, Bagatell R, Weigel BJ, Fox E, Guo D, Van Ryn C, Naranjo A and Park JR: Historical time to disease progression and progression-free survival in patients with recurrent/refractory neuroblastoma treated in the modern era on Children's Oncology Group early-phase trials. Cancer 123: 4914-4923, 2017.

4. The AACR Project GENIE Consortium: AACR Project GENIE: Powering precision medicine through an international consortium. Cancer Discov 7: 818-831, 2017.

5. Trigg RM and Turner SD: ALK in neuroblastoma: Biological and therapeutic implications. Cancers (Basel) 10: 113, 2018.

6. Guan J, Fransson S, Siaw JT, Treis D, Van den Eynden J, Chand D, Umapathy G, Ruuth K, Svenberg P, Wessman S, et al: Clinical response of the novel activating ALK-I1171T mutation in neuroblastoma to the ALK inhibitor ceritinib. Cold Spring Harb Mol Case Stud 4: a002550, 2018.

7. Wesche J, Haglund K and Haugsten EM: Fibroblast growth factors and their receptors in cancer. Biochem J 437: 199-213, 2011.

8. Parker BC, Engels M, Annala M and Zhang W: Emergence of FGFR family gene fusions as therapeutic targets in a wide spectrum of solid tumours. J Pathol 232: 4-15, 2014.

9. Vaughan L, Clarke PA, Barker K, Chanthery Y, Gustafson CW, Tucker E, Renshaw J, Raynaud F, Li X, Burke R, et al: Inhibition of mTOR-kinase destabilizes MYCN and is a potential therapy for MYCN-dependent tumors. Oncotarget 7: 57525-57544, 2016.

10. Chesler L, Schlieve C, Goldenberg DD, Kenney A, Kim G, McMillan A, Matthay KK, Rowitch D and Weiss WA: Inhibition of phosphatidylinositol 3-kinase destabilizes Mycn protein and blocks malignant progression in neuroblastoma. Cancer Res 66: 8139-8146, 2006.

11. Segerström L, Baryawno N, Sveinbjörnsson B, Wickström M, Elfman L, Kogner P and Johnsen JI: Effects of small molecule inhibitors of PI3K/Akt/mTOR signaling on neuroblastoma growth in vitro and in vivo. Int J Cancer 129: 2958-2965, 2011.

12. Klempner SJ, Myers AP and Cantley LC: What a tangled web we weave: Emerging resistance mechanisms to inhibition of the phosphoinositide 3-kinase pathway. Cancer Discov 3: 1345-1354, 2013.

13. Yang J, Nie J, Ma X, Wei Y, Peng Y and Wei X: Targeting PI3K in cancer: Mechanisms and advances in clinical trials. Mol Cancer 18: 26, 2019.

14. Kostopoulou ON, Holzhauser S, Lange BKA, Ohmayer A, Andonova T, Bersani C, Wickström M and Dalianis T: Studies of FGFR3 and PIK3CA mutations in neuroblastomas and the effects of the corresponding inhibitors in neuroblastoma cell lines. Int J Oncol 55: 1372-1384, 2019.

15. Biedler JL, Helson L and Spengler BA: Morphology and growth, tumorigenicity, and cytogenetics of human neuroblastoma cells in continuous culture. Cancer Res 33: 2643-2652, 1973.

16. Biedler JL and Spengler BA: A novel chromosome abnormality in human neuroblastoma and antifolate-resistant Chines hamster cell lives in culture. J Natl Cancer Inst 57: 683-695, 1976.

17. Sugimoto T, Tatsumi E, Kemshead JT, Helson L, Green AA and Minowada J: Determination of cell surface membrane antigens common to both human neuroblastoma and leukemia-lymphoma cell lines by a panel of 38 monoclonal antibodies. J Natl Cancer Inst 73: 51-57, 1984.

18. Helson L and Helson C: Human neuroblastoma cells and 13-cis-retinoic acid. Neurooncol 3: 39-41, 1985. 
19. LaQuaglia MP, Kopp EB, Spengler BA, Meyers MB and Biedler JL: Multidrug resistance in human neuroblastoma cells. J Pediatr Surg 26: 1107-1112, 1991.

20. Holzhauser S, Lukoseviciute M, Andonova T, Ursu RG, Dalianis T, Wickström M and Kostopoulou ON: Targeting fibroblast growth factor receptor (FGFR) and phosphoinositide 3-kinase (PI3K) signaling pathways in medulloblastoma cell lines. Anticancer Res 40: 53-66, 2020.

21. André F, Ciruelos E, Rubovszky G, Campone M, Loibl S, Rugo HS, Iwata H, Conte P, Mayer IA, Kaufman B, et al: Alpelisib for PIK3CA-mutated, hormone receptor-positive advanced breast cancer. N Engl J Med 380: 1929-1940, 2019.

22. Bahleda R, Italiano A, Hierro C, Mita A, Cervantes A, Chan N, Awad M, Calvo E, Moreno V, Govindan R, et al: Multicenter phase I study of erdafitinib (JNJ-42756493), Oral Pan-fibroblast growth factor receptor inhibitor, in patients with advanced or refractory solid tumors. Clin Cancer Res 25: 4888-4897, 2019.

23. Foucquier J and Guedj M: Analysis of drug combinations: Current methodological landscape. Pharmacol Res Perspect 3: e00149, 2015 .

24. Chou TC: Drug combination studies and their synergy quantification using the Chou-Talalay method. Cancer Res 70: 440-446, 2010

25. Holzhauser S, Kostopoulou ON, Ohmayer A, Lange BKA, Ramqvist T, Andonova T, Bersani C, Wickström $\mathrm{M}$ and Dalianis T: In vitro antitumor effects of FGFR and PI3K inhibitors on human papillomavirus positive and negative tonsillar and base of tongue cancer cell lines. Oncol Lett 18: 6249-6260, 2019.

26. Wang L, Suštić T, Leite de Oliveira R, Lieftink C, Halonen P, van de Ven M, Beijersbergen RL, van den Heuvel MM, Bernards R and van der Heijden MS: A functional genetic screen identifies the phosphinositide 3-kinase pathway as a determinant of resistance to fibroblast grown factor receptor inhibitors in FGFR mutant urothelial cell carcinoma. Eur Urol 71: 858-862, 2017.

27. Wang J, Mikse O, Liao RG, Li Y, Tan L, Janne PA, Gray NS Wong KK and Hammerman PS: Ligand-associated ERBB2/3 activation confers acquired resistance to FGFR inhibition in FGFR3-dependent cancer cells. Oncogene 34: 2167-2177, 2015.

28. Herrera-Abreu MT, Pearson A, Campbell J, Shnyder SD, Knowles MA, Ashworth A and Turner NC: Parallel RNA interference screens identify EGFR activation as an escape mechanism in FGFR3-mutant cancer. Cancer Discov 3: 1058-1071, 2013.

29. Brands RC, Knierim LM, De Donno F, Steinacker V, Hartmann S, Seher A, Kübler AC and Müller-Richter UDA: Targeting VEGFR and FGFR in head and neck squamous cell carcinoma in vitro. Oncol Rep 38: 1877-1885, 2017.

30. Singleton KR, Hinz TK, Kleczko EK, Marek LA, Kwak J, Harp T, Kim J, Tan AC and Heasley LE: Kinome RNAi screens reveal synergistic targeting of MTOR and FGFR1 pathways for treatment of lung cancer and HNSCC. Cancer Res 75: 4398-4406, 2015 .
31. Cai W, Song B and Ai H: Combined inhibition of FGFR and mTOR pathways is effective in suppressing ovarian cancer. Am J Transl Res 11: 1616-1625, 2019.

32. Munster P, Aggarwal R, Hong D, Schellens JH, van der Noll R, Specht J, Witteveen PO, Werner TL, Dees EC, Bergsland E, et al: First-in-Human phase I study of GSK2126458, an Oral Pan-class I Phosphatidylinositol-3-Kinase inhibitor, in patients with advanced solid tumor malignancies. Clin Cancer Res 22: 1932-1939, 2016

33. Tamura R, Yoshihara K, Saito T, Ishimura R, Martínez-Ledesma JE, Xin H, Ishiguro T, Mori Y, Yamawaki K, Suda K, et al: Novel therapeutic strategy for cervical cancer harboring FGFR3-TACC3 fusions. Oncogenesis 7: 4, 2018.

34. Fulda S, Honer M, Menke-Moellers I and Berthold F: Antiproliferative potential of cytostatic drugs on neuroblastoma cells in vitro. Eur J Cancer 31A: 616-621, 1995.

35. Zheng X, Naiditch J, Czurylo M, Jie C, Lautz T, Clark S, Jafari N, Qiu Y, Chu F and Madonna MB: Differential effect of long-term drug selection with doxorubicin and vorinostat on neuroblastoma cells with cancer stem cell characteristics. Cell Death Dis 4: e740, 2013.

36. Eschenburg G, Luckert $\mathrm{C}$, Reinshagen $\mathrm{K}$ and Bergholz R: Taurolidine cooperates with antineoplastic drugs in neuroblastoma cells Genes Cancer 5: 460-469, 2014.

37. Wickström M, Johnsen JI, Ponthan F, Segerström L, Sveinbjörnsson B, Lindskog M, Lövborg H, Viktorsson K, Lewensohn R, Kogner P, et al: The novel melphalan prodrug J1 inhibits neuroblastoma growth in vitro and in vivo. Mol Cancer Ther 6: 2409-2417, 2007.

38. Wong $\mathrm{CH}$, Ma BB, Cheong HT, Hui CW, Hui EP and Chan AT: Preclinical evaluation of PI3K inhibitor BYL719 as a single agent and its synergism in combination with cisplatin or MEK inhibitor in nasopharyngeal carcinoma (NPC). Am J Cancer Res 5: 1496-1506, 2015.

39. Keam B, Kim S, Ahn YO, Kim TM, Lee SH, Kim DW and Heo DS: In vitro anticancer activity of PI3K alpha selective inhibitor BYL719 in head and neck cancer. Anticancer Res 35: 175-182, 2015.

40. Konstantinopoulos PA, Barry WT, Birrer M, Westin SN, Cadoo KA, Shapiro GI, Mayer EL, O'Cearbhaill RE, Coleman RL, Kochupurakkal B, et al: Olaparib and $\alpha$-specific PI3K inhibitor alpelisib for patients with epithelial ovarian cancer: A dose-escalation and dose-expansion phase 1b trial. Lancet Oncol 20: 570-580, 2019.

This work is licensed under a Creative Commons Attribution-NonCommercial-NoDerivatives 4.0 International (CC BY-NC-ND 4.0) License. 